Draft version November 10, 2018

Preprint typeset using $\mathrm{LATE}_{\mathrm{E} X} \mathrm{X}$ style emulateapj v. 12/16/11

\title{
FORECASTING THE CONTRIBUTION OF POLARIZED EXTRAGALACTIC RADIO SOURCES IN CMB OBSERVATIONS
}

\author{
G. Puglisi ${ }^{1,2}$, V. Galluzzi ${ }^{3,5}$, L. Bonavera ${ }^{4}$, J. Gonzalez-Nuevo ${ }^{4}$, A. Lapi ${ }^{1}$, M. Massardi $^{3,6}$, F. Perrotta $^{1}$, C. \\ Baccigalupi $^{1,2}$, A. Celotti ${ }^{1,2,7}$, L. DAnese $^{1}$ \\ ${ }^{1}$ SISSA- International School for Advanced Studies, Via Bonomea 265, 34136 Trieste, Italy \\ ${ }^{2}$ INFN-National Institute for Nuclear Physics, Via Valerio 2, I-34127 Trieste, Italy \\ ${ }^{3}$ INAF, Istituto di Radioastronomia, Via Piero Gobetti 101, I-40129 Bologna, Italy \\ ${ }^{4}$ Departamento de Física, Universidad de Oviedo, C. Federico García Lorca 18, 33007 Oviedo, Spain \\ ${ }^{5}$ Dipartimento di Fisica e Astronomia, Università di Bologna, via Gobetti 93/2, I-40129 Bologna, Italy \\ ${ }^{6}$ Italian Alma Regional Centre, Istituto di Radioastronomia, Via Piero Gobetti 101, I-40129 Bologna, Italy and \\ ${ }^{7}$ INAF, Osservatorio Astronomico di Brera, via Bianchi 46, 23807 Merate (LC)
}

(Received November 10, 2018; Revised November 10, 2018; Accepted November 10, 2018)

Draft version November 10, 2018

\begin{abstract}
We combine the latest datasets obtained with different surveys to study the frequency dependence of polarized emission coming from Extragalactic Radio Sources (ERS). We consider data over a very wide frequency range starting from $1.4 \mathrm{GHz}$ up to $217 \mathrm{GHz}$. This range is particularly interesting since it overlaps the frequencies of the current and forthcoming Cosmic Microwave Background (CMB) experiments. Current data suggest that at high radio frequencies, $(\nu \geq 20 \mathrm{GHz})$ the fractional polarization of ERS does not depend on the total flux density. Conversely, recent datasets indicate a moderate increase of polarization fraction as a function of frequency, physically motivated by the fact that Faraday depolarization is expected to be less relevant at high radio-frequencies. We compute ERS number counts using updated models based on recent data, and we forecast the contribution of unresolved ERS in CMB polarization spectra. Given the expected sensitivities and the observational patch sizes of forthcoming CMB experiments about $\sim 200$ ( up to $\sim 2000$ ) polarized ERS are expected to be detected. Finally, we assess that polarized ERS can contaminate the cosmological B-mode polarization if the tensor-to-scalar ratio is $<0.05$ and they have to be robustly controlled to de-lens CMB B-modes at the arcminute angular scales.
\end{abstract}

Subject headings: Cosmology: Cosmic Microwave Background - Radio Sources- observations

\section{INTRODUCTION}

The Cosmic Microwave Background (CMB) is a relic radiation generated at the decoupling of matter and radiation as the temperature of the Universe dropped below $3000 \mathrm{~K}$. Its temperature and polarization anisotropies can be exploited to probe the early stages of the Universe when an exponential expansion, the so called inflation might have occurred (Guth 1981; Starobinsky 1982).

Since last decades, several experiments have tried to measure the CMB polarized signal in order to find the imprints on its polarized anisotropies of a stochastic background of primordial gravitational waves (PGW) that might have been produced during the inflationary phase. Polarization anisotropies are commonly decomposed into two scalar quantities called $E$ - and B-modes (Seljak \& Zaldarriaga 1997; Hu \& White 1997), and to date, lots of efforts have been made to observe the latter since their amplitude at degree scale is expected to come mainly from PGW.

On one hand, E-mode photons get deflected via gravitational interaction by intervening matter of large scale structures during the path toward us, producing the so called lensing B-modes at arcminute scale. Lensing Bmodes have been observed since four years (The Polarbear Collaboration: P. A. R. Ade et al. 2014 Louis et al. 2017; Keisler et al. [2015; The Polarbear Collaboration et al. 2017) with better and better accuracy and they rep-

Corresponding authors: Giuseppe Puglisi, giuspugl@sissa.it resent a powerful tool to probe the large scale structure of our Universe. On the contrary, the primordial B-mode amplitude is unknown and is quantified by the tensor-toscalar ratio, $r$, that relates the amplitude of tensor perturbations of the space time metric, e.g. PGW, with respect to the scalar perturbations. The joint collaboration of BICEP2 and Planck yielded so far the latest upper limit on $r<0.07$ at $95 \%$ confidence level (BICEP2/Keck and Planck Collaborations et al. 2015). Meaning that the primordial B-mode amplitude could be even lower than the lensing one.

To date, several challenges have prevented to detect primordial B-modes mostly because of the diffuse polarized radiation coming from the Milky Way, known as Galactic Foregrounds. The list of Galactic foregrounds is long and includes anything emitting at sub-millimeter wavelengths between us and the CMB: thermal dust, synchrotron radiation, free-free and several molecular line emissions (Planck Collaboration et al. 2016b). All these emissions are partially polarized and the main contribution comes from synchrotron and dust (both polarized up to $20 \%$ level Planck Collaboration et al. 2016e d). At high-frequency $(\nu>90 \mathrm{GHz})$, such a large polarization degree is produced by thermal dust grains aligning along the Galactic magnetic field lines. At low frequencies $(\nu \lesssim 70 \mathrm{GHz})$, cosmic electrons spiralling into the Galactic magnetic field produce synchrotron radiation. Molecular lines are expected to be polarized at lower levels $\lesssim 1 \%$ (Goldreich \& Kylafis 1981; Puglisi et al. 2017), 
whereas free-free emission can be essentially considered unpolarized. This is the justification of the recent efforts aimed at observing the CMB polarization in a very wide range of frequencies and at accurately characterizing both the spatial and frequency distribution of each Galactic polarized foreground. Moreover, such an investigation allows to design algorithms known as component separation or foreground cleaning techniques to extract B-modes out of a multi-frequency experimental setup.

For these reasons, (i) more focal plane pixels in multiple telescopes are needed to increase sensitivity and (ii) multi-band polarization measurements are required to recover the cosmic signal from the Galactic one via component separation. As the focal plane will encode larger and larger number of detectors, the next stages in CMB experiment sensitivity will be achieved by more accurately measuring $r$. To date, several ground based experiments are updating their focal planes to a step forward from the so called CMB-Stage 2 (CMB-S2) to Stage 3 (CMBS3 Arnold et al. 2014; Henderson et al. 2016; Benson et al. 2014), including up to 10,000 detectors observing up to $7 \%$ of the sky. The ultimate step for a B-mode detection from the ground is represented by CMBStage 4 experiments (CMB-S4 Abazajian et al. 2016), which will account for up to 100,000 detectors, observing half of the sky. CMB-S4 aims at measuring $r$ with the target accuracy $\sigma(r) \sim 0.0005$.

At smaller scales the Extragalactic Radio Sources (ERS) and star-forming dusty galaxies are the major contaminants (Tucci et al. 2011), although the latter can also largely contribute to large angular scales due to clustering (De Zotti et al. 2015). In this work, we mostly focus on the polarized emission of ERS. To date, a few studies have been conducted regarding polarization of ERS at the frequencies of CMB experiments (see Galluzzi \& Massardi (2016) or Bonavera et al. (2017a)) since polarization observations in the $\mathrm{mm}$ wavelength bands are more challenging than at $\mathrm{cm}$ bands (at $1.4 \div 20 \mathrm{GHz}$ ) and extrapolations are very common in this field of research (Tucci \& Toffolatti 2012).

The mechanism behind the polarized emission of radio sources is mostly due to synchrotron radiation sourced by an Active Galactic Nucleus (AGN), where a central super-massive black hole $\left(10^{6} \div 10^{9} M_{\odot}\right)$ is hosted. Most of the energy of an AGN comes from the gravitational potential energy of the material located in a thin surrounding accretion disc, released as the matter falls into the central black hole. Another component is constituted by jets (usually paired) of material ejected toward the polar directions from the black hole. Jets are observed to be very collimated and can travel very large distances. Therefore, radio-galaxies seldom present double structures referred as lobes constantly fed by the jets of new energetic particles and magnetic energy.

Depending on which components dominates the emission, such complex objects can obviously appear with different morphologies and therefore be grouped in different observational categories. One of the most important distinction is related to the different orientations an AGN can be observed with respect to the line of sight (see De Zotti et al. (2010) for a wide review). If edgeon, the torus obscures the core and the inner disc, so that the emission is dominated by the optically thin ra- dio lobes presenting a steep spectral index $\alpha$ at low frequencies $1 \div 5 \mathrm{GH}{ }^{1}$ Objects with $\alpha>0.5$ are commonly referred as Steep Spectrum Radio Quasars (SSRQs) and, generally, their optical counterpart is an elliptical galaxy. If seen pole-on, the brightness is dominated by the approaching jet, the emission looks compact and it is mostly Doppler boosted since particles move at relativistic speeds. The emission is optically thick, does not contain many optical features in the continuum but is characterized by a flat spectrum $(\alpha<0.5)$. Similar sources are called Flat Spectrum Radio Quasars (FSRQs).

However, each source presents both the components, i.e. a flat-spectrum core and extended steep-spectrum lobes, and it can be easily understood that a simple-power law cannot be applied to resemble the large radio frequency range (Massardi et al. 2011; Bonaldi et al. 2013). External and self-absorption, from free-free and synchrotron, may affect and change the dependence of $S_{\nu}$, so that the spectrum could increase as a function of frequency (Galluzzi et al. 2017).

There is an increasing interest on polarization of ERS at high-radio frequencies not only to better understand the physics behind the emitting system, e.g. the degree of ordering of the magnetic field, the direction of its field lines (Tucci et al. 2011), but also because polarized ERS will be largely detected by forthcoming CMB experiments. Furthermore, the ERS contaminating signal in the polarization power spectra cannot be neglected to assess the power spectrum of lensing B-modes. This is the reason why recent works in the literature can be found addressing this issue: De Zotti et al. (2015, 2016) predicted the contribution in polarization both for ERS and dusty galaxies at frequency channels of the Cosmic ORigin Explorer (CORE) satellite; Curto et al. (2013) estimated for future $\mathrm{CMB}$ missions the contamination produced by radio and far-Infrared sources at the level of bispectrum considering different shapes of the primordial non-Gaussianity parameter, $f_{n l}$.

In section 2 we describe the datasets we combine in order to determine the polarization dependence as a function of frequency, discussed in section 4 . In section 3 . we present the models for number counts adopted in this analysis. In section 5, we show the results of a forecast package we developed to assess the contamination of polarized ERS in terms of CMB power spectra given the nominal specifics of current and forthcoming CMB experiments. Finally, we devote section 6 to discuss and summarize our results.

\section{DATA}

In this section, we present the data collected from publicly available catalogues. The data, summarized in Table 1. have been used to characterize the polarization fraction of ERS in about two orders of magnitude in frequency range (i.e. from 1.4 to $217 \mathrm{GHz}$ ).

\subsection{The S-PASS/NVSS joint catalogue}

The S-band Polarization All Sky Survey (S-PASS) survey observed the Southern sky with declination $\delta<-1^{\circ}$ at

\footnotetext{
1 The radio-source flux is described by a power law $S_{\nu} \propto \nu^{-\alpha}$, and the threshold between flat and steep spectral behaviour is commonly fixed at $\alpha=0.5$.
} 
TABLE 1

Summary of the catalogues that we use Sec 5

\begin{tabular}{lcccccc}
\hline & Frequency $[\mathrm{GHz}]$ & Sky Region & FWHM & Detect. flux & $90 \%$ Compl. & \# Sources \\
\hline NVSS & 1.4 & $\delta>-40^{\circ}$ & $45^{\prime \prime}$ & $0.29 \mathrm{mJy} / \mathrm{beam}$ & $2.3 \mathrm{mJy}$ & $1.8 \times 10^{6}$ \\
S-PASS & 2.3 & $\delta<-1^{\circ}$ & $8.9^{\prime}$ & $1 \mathrm{mJy} / \mathrm{beam}$ & $420 \mathrm{mJy}$ & 533 \\
JVAS & 8.4 & $\delta \geq 0^{\circ},|b| \geq 2.5^{\circ}$ & $0.2^{\prime \prime}$ & $50 \mathrm{mJy}$ & $200 \mathrm{mJy}$ & 2720 \\
CLASS & 8.4 & $0 \geq \delta \geq 70^{\circ}$ & $0.2^{\prime \prime}$ & $20 \mathrm{mJy}$ & $30 \mathrm{mJy}$ & 16503 \\
AT20G & $4.8,8.6,20$ & $\delta<0^{\circ},|b|<1.5^{\circ}$ & $10^{\prime \prime}, 6^{\prime \prime}, 11^{\prime \prime}$ & $40 \mathrm{mJy}$ & $100 \mathrm{mJy} / \mathrm{beam}$ & 5890 \\
VLA & $4.8,8.5$, & $\delta>-15^{\circ}$ & $12^{\prime \prime}, 6^{\prime \prime}$, & $0.7,0.3$, & $40 \mathrm{mJy}$ & 159 \\
PACO & $22.5,43.5$ & & $4^{\prime \prime}, 2^{\prime \prime}$ & $0.9,1.2 \mathrm{mJy} / \mathrm{beam}$ & & \\
XPOL-IRAM & 20 & Ecl. lat.<-65 & $11^{\prime \prime}$ & $40 \mathrm{mJy}$ & $200 \mathrm{mJy}$ & 104 \\
& 86 & $\delta>30^{\circ}$ & $28^{\prime \prime}$ & $0.5 \mathrm{Jy}$ & $1 \mathrm{Jy}$ & 145 \\
PCCS2 & 30,44, & & $32.4^{\prime}, 27.1^{\prime}$, & 117,229, & 427,692, & 1560,934, \\
& 70,100, & Full sky & $13.3^{\prime}, 9.7^{\prime}$, & 225,106, & 501,269, & 1296,1742, \\
& 143,217 & & $7.3^{\prime}, 5.0^{\prime}$ & $75,81 \mathrm{mJy}$ & $177,152 \mathrm{mJy}$ & 2160,2135 \\
\hline
\end{tabular}

$2.3 \mathrm{GHz}$ with full width at half maximum (FWHM) of 8.9 arcmin both in total intensity and polarization using the $64 \mathrm{~m}$ Parkes Radio Telescope. Lamee et al. (2016) crossmatched it with the NRAO/VLA Sky Survey, Condon et al. (NVSS 1998), at $1.4 \mathrm{GHz}$ (45 arcsec (FWHM) and rms total brightness fluctuations of $\sim 0.29$ mJy beam $^{-1}$ ). Lamee et al. (2016) aimed at generating a novel and independent polarization catalogue ${ }^{2}$ enclosing 533 bright ERS at $2.3 \mathrm{GHz}$ with polarized flux-density stronger than $420 \mathrm{mJy}$.

\subsection{The JVAS/CLASS 8.4 GHz catalogue}

We used the data from the JVAS/CLASS 8.4-GHz catalogue Jackson et al. (2007) $)^{3}$, which combined data taken from the Jodrell-VLA Astrometric Survey (JVAS) and the Cosmic Lens All-Sky Survey (CLASS) both observing at $8.4 \mathrm{GHz}$. The former detected 2720 sources stronger than $200 \mathrm{mJy}$ in total intensity at $5 \mathrm{GHz}$ and $\delta \geq 0^{\circ}$, masking the Galactic mid-plane at Galactic latitude $|b| \geq 2.5^{\circ}$. To complement JVAS, CLASS consisted of all sources with a fainter $5 \mathrm{GHz}$ flux, i.e. $S>30 \mathrm{mJy}$ observed in a sky region between $0^{\circ} \leq \delta \leq 70^{\circ}$. Combining the two surveys, a sample of 16503 FSRQ intensity fluxes has been collected.

Jackson et al. (2010) were able to assess polarized fluxes only for a few objects from the 133 sources observed by WMAP at 22 and $43 \mathrm{GHz}$ Wright et al. $(S>1$ Jy 2009) with counterpart in the JVAS/CLASS catalogues. For the purposes of our work this sample was not large enough to be included in the following analysis.

However, we exploit the data selection described by Pelgrims \& Hutsemékers (2015) that considered all the sources with polarized flux $\geq 1 \mathrm{mJy}$ in order to obtain an unbiased sample of 3858 NED identified sources. We selected 2829 sources classified by Pelgrims \& Hutsemékers (2015) as QSOs and Radio Sources. For a complete description of the catalogue and the surveys refer to Jackson et al. (2007).

\subsection{The AT20G Survey}

The Australia Telescope $20 \mathrm{GHz}$ (AT20G) Survey observed blindly the Southern sky $\left(\delta<0^{\circ}\right.$ excluding the

\footnotetext{
2 http://vizier.cfa.harvard.edu/viz-bin/VizieR?-source= $\mathrm{J} / \mathrm{ApJ} / 829 / 5$

${ }^{3}$ http://vizier.cfa.harvard.edu/viz-bin/VizieR?-source= J/MNRAS/376/371
}

Galactic plane strip at $\left.|b|<1.5^{\circ}\right)$ at $20 \mathrm{GHz}$ with the Australia Telescope Compact Array (ATCA) from 2004 to 2009, (Murphy et al.|2010). The detected sources were followed up almost simultaneously at 4.8 and $8.6 \mathrm{GHz}$. The AT20G source catalogu ${ }^{4}$ includes 5890 sources at $20 \mathrm{GHz}$ above the total intensity detection limit of 40 mJy, of which 3332 were detected at all the observing frequencies. Averaged on the whole area of the survey, the catalogue is $91 \%$ complete above $100 \mathrm{mJy}^{\text {beam }}{ }^{-1}$ (Murphy et al. 2010). Polarization of sources was considered detected if the following criteria were satisfied: polarized flux density $P>6$ mJy or at least three times larger than its rms error, and polarized fraction above 1 per cent. Massardi et al. (2011) presented an analysis to characterize the radio spectral properties of the whole sample both in total intensity and polarization, involving 768 sources detected at $20 \mathrm{GHz}$ (467 of them were also detected in polarization at 4.8 and/or at $8.6 \mathrm{GHz}$ ). Given the goal of this work, we include polarized flux densities from 3332 sources, 2444 of them presenting a flat spectrum in total intensity, $\left|\alpha_{5}^{8}\right|<0.5$ and the remaining 888 a steep-spectrum sources $\left(\left|\alpha_{5}^{8}\right|>0.5\right)$.

\subsection{The VLA observations}

Sajina et al. (2011) presented measurement ${ }^{5}$ in flux densities and polarization of 159 ERSs detected with the Very Large Array (VLA) at four frequency channels, 4.86, 8.46, 22.46, 43.34 GHz. This sample was selected from the AT20G one (Murphy et al. 2010; Massardi et al. 2011) by requiring a flux density $S>40 \mathrm{mJy}$ in the equatorial field of the Atacama Cosmology Telescope (ACT) survey on a region at declination north of $-15^{\circ}$ and excluding the Galactic plane. The aim of this program was firstly to characterize the spectra and variability both in total intensity and polarization of highfrequency-selected radio sources and to improve the estimation of the ERS contamination at high-frequency for CMB experiments.

In $40 \%$ of the whole sample they detected polarized flux density in all the bands, and observed an increasing trend of polarization fraction as a function of frequency, more evident for SSRQs.

\subsection{PACO with ATCA and ALMA}

4 http://vizier.cfa.harvard.edu/viz-bin/VizieR?-source= J/MNRAS/402/2403

5 http://vizier.u-strasbg.fr/viz-bin/VizieR?-source=J/ ApJ/732/45 
The Planck-ATCA Coeval Observations (PACO) project detected 464 sources selected from the AT20G catalogue during 65 epochs between July 2009 and August 2010, at frequencies ranging from 5.5 to $39 \mathrm{GHz}$ with the ATCA. The sources were simultaneously observed (within 10 days) by the Planck satellite (Massardi et al. 2011, Bonavera et al. 2011). The project aimed at characterizing, together with Planck data, the variability and spectral behavior of sources over a wide frequency range (up to $857 \mathrm{GHz}$ for some sources), in total intensity only. The catalogue includes a complete sample of 159 sources selected to be brighter than $200 \mathrm{mJy}$ at $\delta<30^{\circ}$ (excluding the Galactic midplane $|b|<5^{\circ}$ ). A sub-sample of 104 of these sources with ecliptic latitude $<-65^{\circ}$ (which coincides to one of the deep patches most frequently scanned by the Planck satellite scanning strategy) has been re-observed with high sensitivity in polarization with ATCA in 2014 and 2016 in the $1.1-39$ $\mathrm{GHz}$ frequency range (Galluzzi et al. 2017). 32 of them have been also followed up at $95 \mathrm{GHz}$ onto 3 circular regions $\left(10^{\circ}\right.$ of diameter $)$ at ecliptic latitude $<-75^{\circ}$ with the Atacama Large Millimeter Array (ALMA) to better characterize the polarization properties of ERS at the frequencies of many $\mathrm{CMB}$ experiments and allowing an accurate study of few reference targets which could be exploited for calibration and validation of cosmological results. Further details will be described in a companion paper (Galluzzi et al. 2018, in prep.). Data from both 20 and $95 \mathrm{GHz}$ have been included in this analysis.

\subsection{First $3.5 \mathrm{~mm}$ Polarimetric Survey}

Agudo et al. (2010) presented for the first time polarimetric data at $86 \mathrm{GHz}$ of a sample of 145 flat spectrum radio galaxies at different epochs (from 2005 July to 2009 October $\sqrt{6}$. The measurements have been performed by means of the XPOL polarimeter of the IRAM $30 \mathrm{~m}$ telescope, by selecting the sources observed from 1978 to 1994 at $\delta>30^{\circ}$ whose total intensity was above $\gtrsim 1 \mathrm{Jy}$. They detected above $>3 \sigma$ level $1.5 \%$ linear and $0.3 \%$ circular polarization degree respectively for $76 \%$ and $6 \%$ of the whole sample. Remarkably, they found a factor of $\sim 2$ excess in the polarization fraction at $86 \mathrm{GHz}$ with respect to that measured at $15 \mathrm{GHz}$.

\subsection{The Second Planck Catalogue of Compact Sources}

We exploit data from latest Planck Catalogue of Compact Sources (PCCS2 Planck Collaboration 2015 ${ }^{7}$ including polarimetric detection of sources between 30 and $353 \mathrm{GHz}$ from August 2009 to August 2013. The total intensity $90 \%$ completeness ranges from 177 to $692 \mathrm{mJy}$ in this regime of frequencies, allowing to detect thousands of sources matched both internally (between neighbor Planck channels) and with external catalogues. On the contrary, the instrumental noise in polarization and the presence of polarized Galactic foregrounds limited the number of polarized sources to a few tens (with the exception of the $30 \mathrm{GHz}$ channel where 113 polarized sources were detected).

It is straightforward to state that only sources with high fractional polarization have been detected by Planck

6 http://vizier.u-strasbg.fr/viz-bin/VizieR?-source=J/ ApJS/189/1

http://pla.esac.esa.int/pla/ and thus the statistics of ERS polarization can be biased upward. Bonavera et al. (2017a) recently proposed a methodology to cope with this issue by means of applying a stacking technique to Planck data. They used as main sample the $30 \mathrm{GHz}$ catalogue, consisting of 1560 sources above $S>427 \mathrm{mJy}$ at $90 \%$ completeness level and then followed the sample at higher Planck frequency maps. They further distinguished sources inside and outside the Galactic plane defined by the Planck Galactic mask GAL060 $\left(f_{s k y} \approx 60 \%\right)$ and the exclusion of the Small and Large Magellanic clouds. This technique has been already applied by Stil et al. (2014) to NVSS dataset to study the faint polarized signal of ERS detected in total intensity: the signal from many weak sources is coadded to achieve a statistical detection. Bonavera et al. (2017a) found that the ERS polarization fraction is approximately constant with frequency over the Planck frequency range. An alternative approach that attempts to overcome some of the intrinsic statistical limitations of the stacking technique have been recently exploited by Trombetti et al. (2017) obtaining results comparable both with Bonavera et al. (2017a b) and with other ground based observations.

We used both data coming from the PCCS2 catalogue and from Bonavera et al. (2017a).

\section{MODEL FOR NUMBER COUNTS}

We adopted the evolutionary model proposed by de Zotti et al. (2005, hereafter, D05) that describes the population properties of ERSs and dusty galaxies above $\nu \gtrsim 5 \mathrm{GHz}$. The model assumes a simple analytic luminosity evolution in order to fit the available data on local luminosity functions (LF), source counts 8 and redshift distributions for sources down to few mJy. It determines the epoch-dependent LF starting from local LFs for several source populations. For each population the model adopts a different evolution laws estimating a set of free parameters from available data. Recently, Bonato et al. (2017) and Mancuso et al. (2017) improved the predictions of D05 model by updating the LF and redshift evolution with state of art data of radio-emitting star-forming galaxies and AGNs.

The D05 model assumes a power-law spectrum for each considered population of ERS and each one is described by one (or at most two) constant spectral index. This simple assumptions could not hold anymore when large frequency ranges are taken into account. Departures from single power law spectra are expected because of (i) electron ageing (ii) transition from optically thick to optically thin regime, (iii) different components yielding different spectral contributions at different frequencies. Therefore, this simplified model requires adjusting when source counts measurement are observed at frequencies $>40 \mathrm{GHz}$.

Tucci et al. (2011) showed that radio spectra in AGN cores can differ from a single power-law when large frequency intervals are considered. In particular, they focused on the blazar spectra for which a steepening of the spectral index from 0.5 to 1.2 has been observed (Planck Collaboration et al. 2011ab) due to the transition from optically-thick to optically-thin synchrotron

8 Available online http://w1.ira.inaf.it/rstools/srccnt/ srccnt_tables.html 

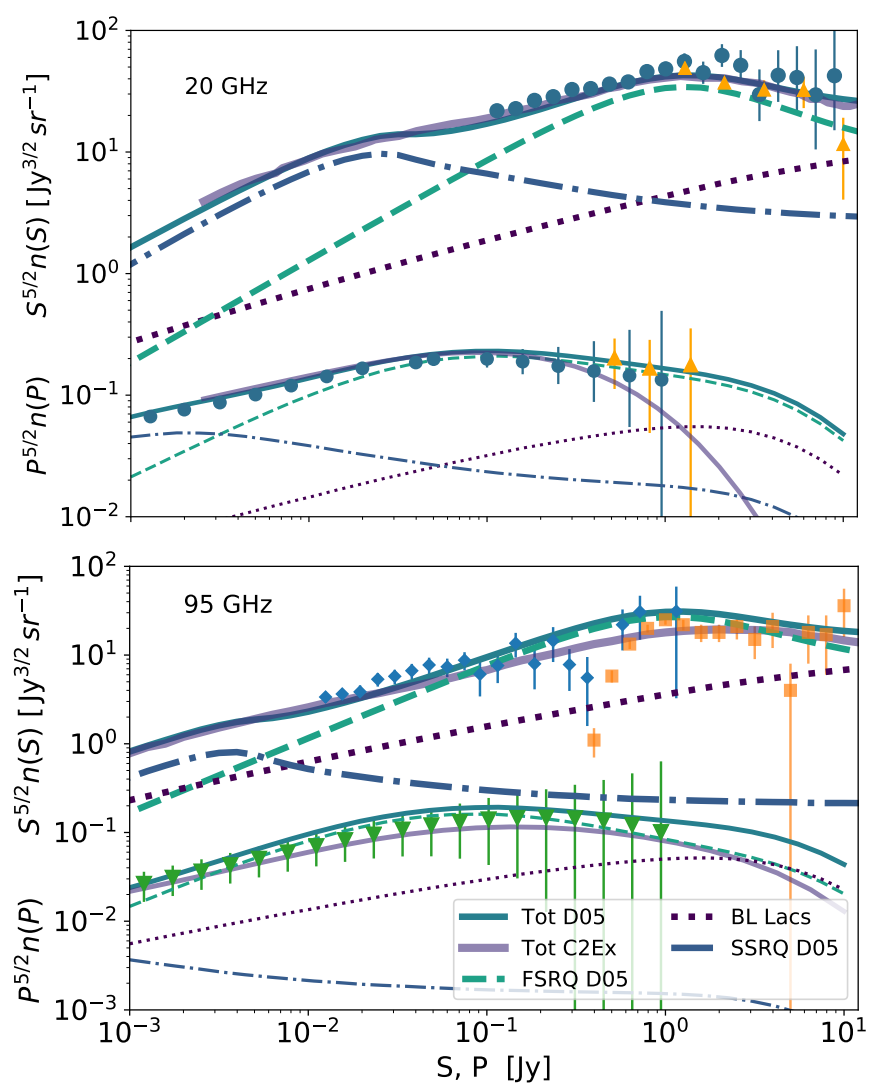

FIG. 1. Euclidean differential number counts at (top) 20 and (bottom) $95 \mathrm{GHz}$. Thick dotted, dashed, dot-dashed and solid lines are respectively the number counts of BL Lacs, FSRQs, SSRQs and their total contribution predicted by the D05 model (de Zotti et al. 2005). The thick solid gray line show the number counts prediction from the C2Ex model (Tucci et al. 2011). Thinner lines follow the same color scheme as the thick ones and refer to polarization number counts, computed via a convolution with a log-normal distribution function fitted from the data. Number counts estimates from several surveys are also shown. (top) The circle data point in the upper curves are data from AT20G (Massardi et al. 2008), whereas upper triangles are from WMAP5-yr survey (K-band $\overline{M a s}-$ sardi et al. 2009); in lower curves polarization number counts trom a resampling of PACO data (Galluzzi et al. 2018, circles) and from WMAP polarization point source catalogue (Lopez-Caniego et al. 2009, , upper triangles). (bottom panel) Diamonds in upper curves are number counts from SPT (Mocanu et al. 2013), squares are from Planck ERCSC catalogue (Planck Collaboration et al. 2011a); the lower triangles have been obtained from a bootstrap resampling of 32 polarized fluxes detected with PACO at $95 \mathrm{GHz}$.

emission of AGN jets (Kellermann 1966; Blandford \& Koenigl 1979). Therefore, Tucci et al. (2011) proposed the so called C2Ex model that assumes a spectral break and different parameters for BL Lacs and FSRQs and allows to properly fit the number counts especially at high-frequency $(\nu \geq 100 \mathrm{GHz})$. Furthermore, Planck Collaboration (2015, XXVI) found that all radio sources observed at the Low Frequency Instrument (LFI) channels present flat and narrow spectral index distribution with $\alpha_{L F I} \lesssim 0.2$, whereas sources in the High Frequency Instrument (HFI) catalogues have a broader distribution showing a steeper spectral index, $\alpha_{H F I} \gtrsim 0.5$ and these findings supports the scenario of BL Lac transition happening at larger frequencies $\nu>100 \mathrm{GHz}$ with respect to the FSRQ one (at $10<\nu<100 \mathrm{GHz}$ ).

We plot in Figure 1 as thicker curves. the differential number counts, $n(S)$, predicted with D05 and
C2Ex models respectively as blue and grey thick solid lines. The top (bottom) panel refers to number counts at $20(95) \mathrm{GH}_{2} 9$. We also plot the contributions estimated by the D05 model for BL Lacs, FSRQs, SSRQs respectively as dotted, dashed, dot-dashed lines. To compare the quantities with those expected in a Euclidean Universe, counts are normalized by a factor of $S^{5 / 2}$. The data points shown are number counts as measured by AT20G survey (Massardi et al. 2008, blue circles), from South Pole Telescope (SPT Vieira et al. 2010; Mocanu et al. 2013, blue diamonds), from the Wilkinson Microwave Anisotropy Probe (WMAP Massardi et al. 2009, yellow upper triangles) and from Planck (Planck Collaboration et al. 2011a, 2013, yellow squares).

The lower thinner curves in Figure 1 are Euclidean normalized differential polarized emission number counts, $P^{5 / 2} n(P)$, computed from polarized flux-density measurements and will be discussed in Sec. 4 .

By comparing the predictions from the two models, we find that both are in a reasonable agreement, with differences well below the uncertainties at $20 \mathrm{GHz}$. However, as discussed above and shown in the bottom panel of Figure 1, number counts estimated with D05 are systematically a factor of $\sim 2$ higher than the C2Ex ones at larger fluxes $100 \mathrm{mJy}$, consistently with the findings of Planck Collaboration et al. (2011a).

In the following, we make use of both D05 and C2Ex models to assess respectively conservative and realistic estimates of polarized ERS to CMB measurements.

\section{STATISTICAL PROPERTIES OF ERS POLARIZATION FRACTION}

Polarization number counts have to be assessed to know how many sources can be detected at a certain polarized flux density, $P=\sqrt{Q^{2}+U^{2}}$, with $Q$ and $U$ being the linear polarization Stokes parameters. Polarization measurements at mm-wavelengths are scarce because of the faintness of the polarized signal, so that both high sensitivity and robust estimates of systematic effects are required. Furthermore, completeness is very hard to be achieved with polarized samples. This is the reason why, to date, extrapolations from low frequency observations $(1.4 \div 5 \mathrm{GHz})$ are commonly adopted though the uncertainties due to intra-beam effects and bandwidth depolarization may seriously affect the estimation.

To encompass this issue, several works in the literature (Battye et al. 2011; Tucci \& Toffolatti 2012; Massardi et al. 2013; Bonavera et al. 2017a) considered the probability function $\mathcal{P}($ II) of the polarization fraction, $\Pi=P / S$. Differential polarization number counts can be defined as

$$
\begin{aligned}
n(P) & =N \int_{S_{0}=P}^{\infty} \mathcal{P}(P, S) d S=N \int_{S_{0}=P}^{\infty} \mathcal{P}(\Pi, S) \frac{d S}{S} \\
& =\int_{S_{0}=P}^{\infty} \mathcal{P}(\Pi) n(S) \frac{d S}{S},
\end{aligned}
$$

where $N$ is the total number of sources with $S \geq S_{0}$, $\mathcal{P}(P, S)$ and $\mathcal{P}(\Pi, S)$ are the probability functions of finding a source with flux $S$ and polarized flux $P$ or polar-

9 Source number counts for a wider range of frequencies are shown in Figure A.1 

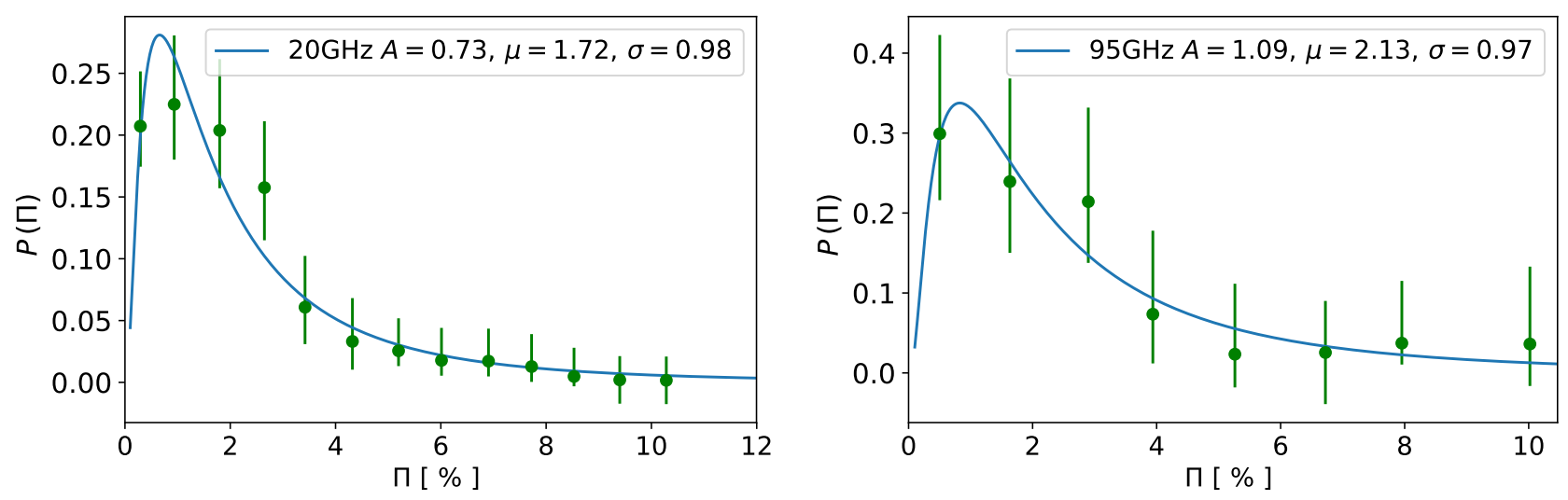

FIG. 2.- Distribution function of polarization fraction for data at $20 \mathrm{GHz}$ (left) and at $95 \mathrm{GHz}$ (right). The best fit values of log-normal parameters are shown. The reduced $\tilde{\chi}^{2}$ estimated from the fit is 0.13 and 0.15 respectively for left and right panels.

ization fraction $\Pi$ and both can be constrained from observations.

Notice that, in the last equation of (1), we assume that $\Pi$ and $S$ are statistically independent. On one hand, recent results at low frequencies indicate that this might not be the case: Stil et al. (2014) found that fainter sources $(\sim 1 \mathrm{mJy})$ of NVSS catalogue present a higher median fractional polarization. These results were confirmed by Lamee et al. (2016) with S-PASS: they found indications of a possible correlation between the polarization fraction and total intensity of steep-spectrum sources ranging from 0.42 to $10 \mathrm{Jy}$, whereas the correlation disappears when FSRQs are involved. On the other hand, at higher frequencies (above $20 \mathrm{GHz}$ ), Massardi et al. (2008) and Tucci \& Toffolatti (2012) did not find a clear correlation between 11 and $S$ (at fluxes above 500 mJy) for both FSRQs and SSRQs, but they found fractional polarization correlating at frequencies between 4.8 and $20 \mathrm{GHz}$.

To date, surveys at high-frequencies have not been sensitive enough to probe fainter polarized fluxes in order to seek whether this assumption holds or not. Tucci et al. (2004) further argued that at higher frequencies we observe two possible effects: (i) depolarization from Faraday rotation is essentially negligible at frequencies above $\nu \gtrsim 10 \mathrm{GHz}$, (ii) by observing compact objects (i.e. FS$\mathrm{RQs}$ ) at increasing frequency, we probe inner and inner regions, closer to the nucleus where the magnetic field is expected to be highly ordered. Consequently if this is the case, the polarization fraction may increase with the frequency.

Given the goals of our work and the fact that frequencies above $10 \mathrm{GHz}$ are involved in the forecast analysis, we assume polarized fraction and flux-density uncorrelated and statistically independent but we look for some eventual dependence of $\Pi$ as function of frequency.

Following Battye et al. (2011), we model $\mathcal{P}(\Pi)$ by means of a log-normal distribution, i.e.

$$
\mathcal{P}(\Pi)=\frac{A}{\sqrt{2 \pi \sigma^{2}} \Pi} \exp \left[-\frac{(\ln (\Pi / \mu))^{2}}{2 \sigma^{2}}\right]
$$

where $\mu$ and $\sigma$ are respectively the median and the standard deviation in log. Notice that eq. (2) holds only if $0 \leq \Pi<\infty$. Although an infinite value of $\Pi$ does not have any physical meaning (synchrotron emission can be polarized up to $75 \%$ ), the values of $\mu$ and $\sigma$ are orders of magnitude smaller. Thus $\Pi$ can be effectively assumed to range up to a large value. This allows us to write a good approximation of the fractional polarization by a combination of the log-normal parameter: 10

$$
\begin{aligned}
\langle\Pi\rangle & \approx \mu e^{\frac{1}{2} \sigma^{2}}, \\
\left\langle\Pi^{2}\right\rangle & \approx \mu^{2} e^{2 \sigma^{2}}, \\
\Pi_{\text {med }} & \approx \mu .
\end{aligned}
$$

We derive polarization fraction distribution by using a bootstrap-resampling method outlined in Austermann et al. (2009). This generates $N_{\text {resamp }}$ simulations of the catalogue and values for unpolarized and polarized flux densities are randomly assigned for each source, from a normal distribution $\mathcal{N}\left(\mu_{s r c}, \sigma_{s r c}\right)$ peaking at the observed value $\mu_{s r c}$ and with a width $\sigma_{s r c}$ equal to the flux uncertainty. In the case of upper limits, a random number is extracted from a normal distribution centred on 0 and with width $\sigma_{s r c}$. For each resampling we compute the polarization fraction and the values are distributed across bins (ranging from 5 to 15 bins depending on the number of data collected in each catalogue). The final distribution is thus given by the mean value within each bin and vertical error bars computed by means of Poisson statistics, at $68 \%$ of confidence level (CL, Gehrels 1986), counting the observed sources in each polarization fraction bin. Finally, a log-normal distribution function (2) is fitted from each dataset and $\langle\Pi\rangle,\left\langle\Pi^{2}\right\rangle$ and $\Pi_{m e d}$ are then estimated from the log-normal parameters $\mu$ and $\sigma$ as in (3), (4), (5). We show in Figure 2 the polarization fraction distributions from PACO-ATCA at $20 \mathrm{GHz}$ and PACOALMA at $95 \mathrm{GHz}$ (the best fit parameters of the other datasets used in this analysis are summarized in Table 2). We show in top panel of Figure 1 the polarization number counts computed by Galluzzi et al. (2018) at 20 $\mathrm{GHz}$ (blue circles) as a result of the convolution of total intensity number counts with the log-normal distribution $\mathcal{P}(\Pi)$ as in eq. (1). We further overlap the predicted total counts from both the D05 (solid thin blue) and C2Ex (solid thin gray) models convolved with the distribution function. As already stated in Sec. 3, at $20 \mathrm{GHz}$ both

\footnotetext{
10 For further details refer to Battye et al. (2011).
} 
Forecasting Polarized Radio Sources for CMB observations

TABLE 2

VALUES OF LOG-NORMAL PARAMETERS OBTAINED By FItTing DATA FROM EACH CATALOGUe.

\begin{tabular}{|c|c|c|c|c|c|c|c|c|}
\hline \multicolumn{9}{|c|}{ Flat-spectrum sources } \\
\hline$\nu[\mathrm{GHz}]$ & $N_{s r c}$ & $A$ & $\mu$ & $\sigma$ & $\langle\Pi\rangle$ & $\Pi_{\text {med }}$ & $\left\langle\Pi^{2}\right\rangle^{1 / 2}$ & Reference \\
\hline 1.4 & 82 & $0.54 \pm 0.08$ & $1.73 \pm 0.24$ & $1.05 \pm 0.09$ & $2.98 \pm 0.64$ & $1.72 \pm 0.24$ & $5.15 \pm 1.53$ & Lamee et al. $(2016)$ \\
\hline 2.3 & 82 & $0.53 \pm 0.07$ & $1.51 \pm 0.13$ & $1.05 \pm 0.07$ & $2.64 \pm 0.36$ & $1.52 \pm 0.13$ & $4.59 \pm 0.87$ & Lamee et al. $(\overline{2016})$ \\
\hline 4.8 & 2335 & $1.57 \pm 0.07$ & $2.36 \pm 0.02$ & $0.75 \pm 0.01$ & $3.14 \pm 0.03$ & $2.37 \pm 0.02$ & $4.16 \pm 0.08$ & Murphy et al. (2010) \\
\hline 8.6 & 2335 & $1.55 \pm 0.02$ & $2.46 \pm 0.01$ & $0.73 \pm 0.01$ & $3.21 \pm 0.03$ & $2.46 \pm 0.01$ & $4.20 \pm 0.06$ & Murphy et al. $(2010)$ \\
\hline 8.6 & 2827 & $0.52 \pm 0.01$ & $2.41 \pm 0.05$ & $0.76 \pm 0.01$ & $3.23 \pm 0.08$ & $2.41 \pm 0.05$ & $4.31 \pm 0.14$ & Pelgrims \& Hutsemékers (2015) \\
\hline 4.8 & 109 & $0.60 \pm 0.06$ & $2.02 \pm 0.13$ & $0.84 \pm 0.05$ & $2.89 \pm 0.28$ & $2.02 \pm 0.13$ & $4.12 \pm 0.55$ & Sajina et al. $(2011)$ \\
\hline 8.6 & 109 & $0.74 \pm 0.14$ & $2.12 \pm 0.24$ & $0.84 \pm 0.09$ & $3.01 \pm 0.51$ & $2.12 \pm 0.23$ & $4.27 \pm 1.02$ & Sajina et al. $(\overline{2011})$ \\
\hline 22 & 155 & $1.36 \pm 0.09$ & $3.1 \pm 0.10$ & $0.88 \pm 0.03$ & $4.57 \pm 0.22$ & $3.10 \pm 0.09$ & $6.74 \pm 0.46$ & Sajina et al. $(2011)$ \\
\hline 43 & 111 & $2.59 \pm 0.08$ & $4.48 \pm 0.06$ & $1.00 \pm 0.03$ & $7.42 \pm 0.17$ & $4.47 \pm 0.06$ & $12.32 \pm 0.41$ & Sajina et al. \\
\hline 20 & 104 & $0.73 \pm 0.05$ & $1.73 \pm 0.16$ & $0.98 \pm 0.06$ & $2.91 \pm 0.42$ & $1.73 \pm 0.16$ & $4.89 \pm 0.99$ & Galluzzi et al. (2018) \\
\hline 89 & 145 & $1.20 \pm 0.06$ & $2.86 \pm 0.10$ & $0.64 \pm 0.03$ & $3.52 \pm 0.17$ & $2.86 \pm 0.10$ & $4.32 \pm 0.28$ & Agudo et al. $(2010)$ \\
\hline 95 & 32 & $1.09 \pm 0.21$ & $2.13 \pm 0.23$ & $0.97 \pm 0.09$ & $3.20 \pm 0.60$ & $2.07 \pm 0.24$ & $4.94 \pm 1.32$ & This work \\
\hline 30 & 114 & $1.51 \pm 0.23$ & $2.05 \pm 0.36$ & $1.08 \pm 0.08$ & $3.69 \pm 0.92$ & $2.06 \pm 0.37$ & $6.61 \pm 2.19$ & Planck Collaboration $(2015)$ \\
\hline 44 & 30 & $2.63 \pm 0.26$ & $2.72 \pm 0.26$ & $0.77 \pm 0.11$ & $3.69 \pm 0.66$ & $2.73 \pm 0.26$ & $5.00 \pm 1.32$ & Planck Collaboration \\
\hline 70 & 34 & $3.91 \pm 0.55$ & $2.52 \pm 0.05$ & $0.58 \pm 0.06$ & $2.97 \pm 0.15$ & $2.51 \pm 0.05$ & $3.52 \pm 0.30$ & Planck Collaboration \\
\hline 100 & 20 & $2.18 \pm 0.28$ & $5.15 \pm 0.69$ & $0.80 \pm 0.10$ & $7.19 \pm 1.59$ & $5.17 \pm 0.73$ & $9.99 \pm 3.07$ & Planck Collaboration $(\overline{2015})$ \\
\hline 143 & 25 & $3.13 \pm 0.10$ & $5.98 \pm 0.16$ & $0.80 \pm 0.04$ & $8.39 \pm 0.35$ & $6.02 \pm 0.13$ & $11.69 \pm 0.80$ & Planck Collaboration \\
\hline 217 & 11 & $3.44 \pm 0.32$ & $3.74 \pm 0.29$ & $0.88 \pm 0.11$ & $5.47 \pm 0.34$ & $3.70 \pm 0.27$ & $8.09 \pm 1.09$ & $\overline{\text { Planck Collaboration }}(\overline{2015})$ \\
\hline \multicolumn{9}{|c|}{ Steep-spectrum sources } \\
\hline 1.4 & 388 & $1.12 \pm 0.08$ & $1.47 \pm 0.11$ & $1.05 \pm 0.08$ & $2.56 \pm 0.35$ & $1.47 \pm 0.11$ & $4.45 \pm 0.95$ & Lamee et al. (2016) \\
\hline 2.3 & 388 & $1.78 \pm 0.07$ & $1.93 \pm 0.06$ & $0.80 \pm 0.05$ & $2.66 \pm 0.14$ & $1.93 \pm 0.06$ & $3.66 \pm 0.31$ & $\overline{\text { Lamee et al. }}(\overline{\overline{2016}})$ \\
\hline 4.8 & 952 & $2.07 \pm 0.07$ & $2.83 \pm 0.07$ & $0.81 \pm 0.04$ & $3.92 \pm 0.15$ & $2.84 \pm 0.08$ & $5.43 \pm 0.37$ & Murphy et al. 2010 \\
\hline 8.4 & 952 & $3.02 \pm 0.03$ & $2.13 \pm 0.12$ & $1.13 \pm 0.03$ & $4.85 \pm 0.05$ & $3.02 \pm 0.05$ & $7.79 \pm 0.18$ & Murphy et al. $(2010)$ \\
\hline 20 & 952 & $4.55 \pm 0.12$ & $6.98 \pm 0.12$ & $0.55 \pm 0.01$ & $8.10 \pm 0.18$ & $6.98 \pm 0.12$ & $9.41 \pm 0.27$ & Murphy et al. $(2010)$ \\
\hline 4.8 & 39 & $2.72 \pm 0.65$ & $2.35 \pm 0.46$ & $1.07 \pm 0.43$ & $4.19 \pm 1.42$ & $2.35 \pm 0.42$ & $7.49 \pm 5.61$ & Sajina et al. (2011) \\
\hline 8.6 & 39 & $1.94 \pm 0.14$ & $3.39 \pm 0.31$ & $1.04 \pm 0.10$ & $5.90 \pm 1.10$ & $3.41 \pm 0.32$ & $10.23 \pm 2.95$ & Sajina et al. $(2011)$ \\
\hline 22 & 38 & $2.51 \pm 0.10$ & $5.76 \pm 0.19$ & $0.82 \pm 0.05$ & $8.08 \pm 0.44$ & $5.73 \pm 0.17$ & $11.40 \pm 0.99$ & Sajina et al. $(\overline{2011})$ \\
\hline 43 & 15 & $4.74 \pm 0.08$ & $9.89 \pm 0.20$ & $0.73 \pm 0.02$ & $12.43 \pm 0.29$ & $9.62 \pm 0.13$ & $16.06 \pm 0.55$ & Sajina et al. 2011$)$ \\
\hline
\end{tabular}

models are equivalent even for polarized number counts.

In bottom panel of Figure 1 are shown the polarized number counts at $95 \mathrm{GHz}$ coming from the PACO-ALMA sample of 32 sources as lower green triangles. Given the paucity of this sample, we re-sample it by means of 1,000 bootstrap-resampling. The resampled source counts (shown as green lower triangles in Figure 1) are then computed in a similar manner as for the $20 \mathrm{GHz}$ observations and are summarized in the companion paper by Galluzzi et al. (2018, in prep.). The error bar estimation of each data point include the Poissonian 68\% CL uncertainties (Gehrels 1986) plus the error derived from the uncertainties of log-normal parameters $\delta_{A}, \delta_{\mu}, \delta_{\sigma}$ (summarized in Table 2). This error has been assessed by means of differencing the number counts convolved with an upper and a lower log-normal function, respectively estimated at maximum and minimum values of log-normal parameters.

We would like to stress that this is the first time that number counts from the PACO-ALMA sample have been computed and exploited for this kind of analysis. Notice that the data are very well fitted by both predictions.

The estimated values of $\langle\Pi\rangle, \Pi_{m e d}$ and $\left\langle\Pi^{2}\right\rangle^{1 / 2}$ for FSRQ(left panel) and SSRQ (right panel) are shown in Figure 3. By comparing the two panels, we note that the SSRQ fractional polarizations increases with frequency. Although this could be simply related to observational bias (at higher frequencies, steep-spectrum sources contributes at fainter fluxes), such frequency dependence of $\Pi$ for SSRQs has been already discussed in Tucci \& Toffo- latti (2012). On the contrary, the fractional polarization measured for the FSRQ remains almost constant during the frequency range studied. To quantify this dependence, we estimate a linear fit on $\left\langle\Pi^{2}\right\rangle^{1 / 2}$ as a function of a wide (around 2 orders of magnitude) range of frequencies. This choice is mainly due to the fact that $\left\langle\Pi^{2}\right\rangle$ values are needed to estimate B-mode angular power spectrum of polarized ERSs and we include in the linear fit also the values of $\left\langle\Pi^{2}\right\rangle^{1 / 2}$ estimated by Bonavera et al. (2017a) between 30 and $217 \mathrm{GHz}$. They were derived assuming a log-normal distribution as in this work. In particular, for the best fit, we retain only fractional polarization from the FSRQs and BL Lacs since their contribution dominates number counts at larger fluxes and at frequencies $>20 \mathrm{GHz}$ (see Figures 1 and A.1). The linear fit involves the data for which the estimation of $\mu$ and $\sigma$ are reliable (filled symbols in Figure 3). Open symbols indicate data that have not been included to the fit, mainly because of the poor statistics in fitting the log-normal distribution (e.g. less than 20 polarized sources have been detected in polarization in the Planck HFI channels, see Table2.

We find a negligible frequency dependence of $\left\langle\Pi^{2}\right\rangle^{1 / 2}$ :

$$
\begin{gathered}
\left\langle\Pi^{2}\right\rangle^{1 / 2}(\nu)=\left(0.005 \pm 0.006 \mathrm{GHz}^{-1}\right) \nu \\
+(4.170 \pm 0.22) .
\end{gathered}
$$

In the top left panel of Figure 3 we show the linear fit as a gray solid line with darker and lighter shaded areas resembling respectively the $1 \sigma$ and $2 \sigma$ uncertainties 
on best fit parameters. Notice that for $\nu>20 \mathrm{GHz}$, we found $\left\langle\Pi^{2}\right\rangle^{1 / 2} \sim 4 \%$, in agreement with the value found by Tucci \& Toffolatti (2012) and consistent with the expectations of Tucci et al. (2004) and Stil et al. (2014).

At $1 \nu<20 \mathrm{GHz}$, SSRQs have to be taken into account to forecast the contribution of ERS to CMB observations. Thus, we perform the same linear fit by including SSRQs for all the datasets at frequencies smaller than $20 \mathrm{GHz}$, shown in Figure 3 (top right panel). The best fit equation changes into

$$
\begin{gathered}
\left.\left\langle\Pi^{2}\right\rangle^{1 / 2}(\nu)=(-0.015 \pm 0.009) \mathrm{GHz}^{-1}\right) \nu \\
+(5.43 \pm 0.23) .
\end{gathered}
$$

Nonetheless the slope is still negligible, the presence of SSRQs enhances the average polarization fraction of sources at frequencies $\nu \lesssim 20 \mathrm{GHz}$ and, as one can notice in Figure 3 , this is consistently observed in $\langle\Pi\rangle$ as well.

We would like to stress that selection effects could bias our results towards larger values of $\Pi$, especially where few tens of polarized sources have been detected, see Table 2. This is the reason why we excluded PCCS2 HFI data (magenta diamonds) in Figure 3 and we considered the ones from Bonavera et al. (2017a) (gray pentagons). To this regard, the stacking technique helps because it includes the faint sources to the statistical estimate of $\Pi$ even if those sources are not directly detectable.

\section{FORECASTS FOR FORTHCOMING CMB GROUND-BASED EXPERIMENT}

In this section we present the forecast analysis for current and forthcoming CMB surveys performed with a Python package Point Source ForeCast (PS4C) made publicly available ${ }^{11}$. PS4C is a user friendly platform which allows to forecast the contribution of radio point sources both in total intensity and polarized fluxdensities given the nominal specifics of a CMB experiment. In Table3 we summarize the specifics of $5 \mathrm{CMB}$ experiments with whom we forecast the ERS contribution with PS4C:

- the Q-U-I JOint TEnerife López-Caniego et al. (QUIJOTE 2014) CMB experiment designed to observe the polarized emissions from the CMB, our Galaxy and the extra-galactic sources at four frequencies in the range between 10 and $20 \mathrm{GHz}$ and at FWHM resolution of $\sim 1^{\circ}$. Observations started observing in November 2012, covering 18, $000 \mathrm{deg}^{2}$ of the Northern hemisphere, and achieved the sensitivity of $1800 \mu \mathrm{K}$ arcmin in polarization;

- a generic CMB-S2 experiment observing at 95, 150 $\mathrm{GHz}$ within a patch including $2 \%$ of the sky at the resolution of 3.5 arcmin, at $25 \div 30 \mu \mathrm{K}$ arcmin sensitivity;

- a CMB-S3 ground based experiment with the socalled strawman configuration, as it has been defined in Abazajian et al. (2016), for the "measuring$r$ " survey. It consists of an array of small-aperture $(\mathrm{SA}, \sim 1 \mathrm{~m}$ ) telescopes and one large-aperture (LA, $\sim 5 \mathrm{~m}$ ) telescope, observing at the accessible atmospheric windows in the sub-millimeter range (at

11 https://gitlab.com/giuse.puglisi/PS4C about $30,40,90,150 \mathrm{GHz})$. The sensitivities at these frequencies are targeted to be about $1 \div 10 \mu \mathrm{K}$ arcmin.

- the Lite satellite for the studies of B-mode polarization and Inflation from cosmic Background Radiation Detection (LiteBIRD Matsumura et al. 2016) is a satellite mission proposed to JAXA aimed at measuring the CMB polarized signal at degree angular scale. Its goal is to characterize the measurement of $r$ with an uncertainty $\sigma(r)<0.001$. In order to achieve such high accuracy, the target detector sensitivity is $2 \mu \mathrm{K}$ arcmin observing over a wide range of frequencies (from 40 to $320 \mathrm{GHz}$ ). The current effort aims to launch in 2025;

- the Cosmic ORigin Explorer Delabrouille et al. 2017. CORE) is a next generation space-borne experiment and it has been proposed as a Mediumsize ESA mission opportunity. It has been designed as the Planck satellite successor, planned to have better angular resolution and sensitivity than Planck. We consider the CORE150 configuration: a satellite involving a $1.5 \mathrm{~m}$ telescope, observing over a wide range of frequency channels (up to $800 \mathrm{GHz}$ ) with sensitivities ranging from $\sim 10$ to $5 \mu \mathrm{K}$ arcmin. In this work, we restrict our analysis to a selection of frequency channels, (see the last row of Table 3) to compare the expectations with the ones previously obtained by De Zotti et al. (2016).

Although most of the frequency channels of future experiments range up to $350 \mathrm{GHz}$, we forecast up to 150 $\mathrm{GHz}$. This is because at higher frequencies the contribution coming from dusty galaxies and Cosmic Infrared Background cannot be neglected ${ }^{12}$ (Negrello et al. 2013 . De Zotti et al. 2016). Bonavera et al. (2017b) estimated the polarized contribution of dusty galaxies by stacking about 4700 sources observed by Planck at 143, 217, 353 $\mathrm{GHz}$ HFI channels. They estimated the polarized contribution of dusty galaxies to B-mode power spectra and found that at frequencies larger than $217 \mathrm{GHz}$ these population of sources might remarkably contaminate the primordial B-modes.

We compute one realization of CMB power spectra by means of the CAMB package (Lewis et al. 2000) by assuming the Planck best fit cosmological parameters (Planck Collaboration et al.2016c) and a tensor to scalar ratio $r=0.05$ (slightly below the current upper limits).

To assess the contribution of ERS to the power spectrum level, we assume their distribution in the sky to be Poissonian, since the contribution of clustering starts to be relevant for $S<10$ mJy (González-Nuevo et al. 2005, Toffolatti et al. 2005). The power spectrum of temperature fluctuations coming from a Poissonian distribution of sources is expected to be a constant contribution at all multipoles. In particular, we consider as masked all sources whose flux-density is above $3 \sigma$ the detection limit

12 We have already planned to include into the package the contribution from dusty galaxies and forecasts with PS4C will be presented in a future release that will be described in a future paper. 

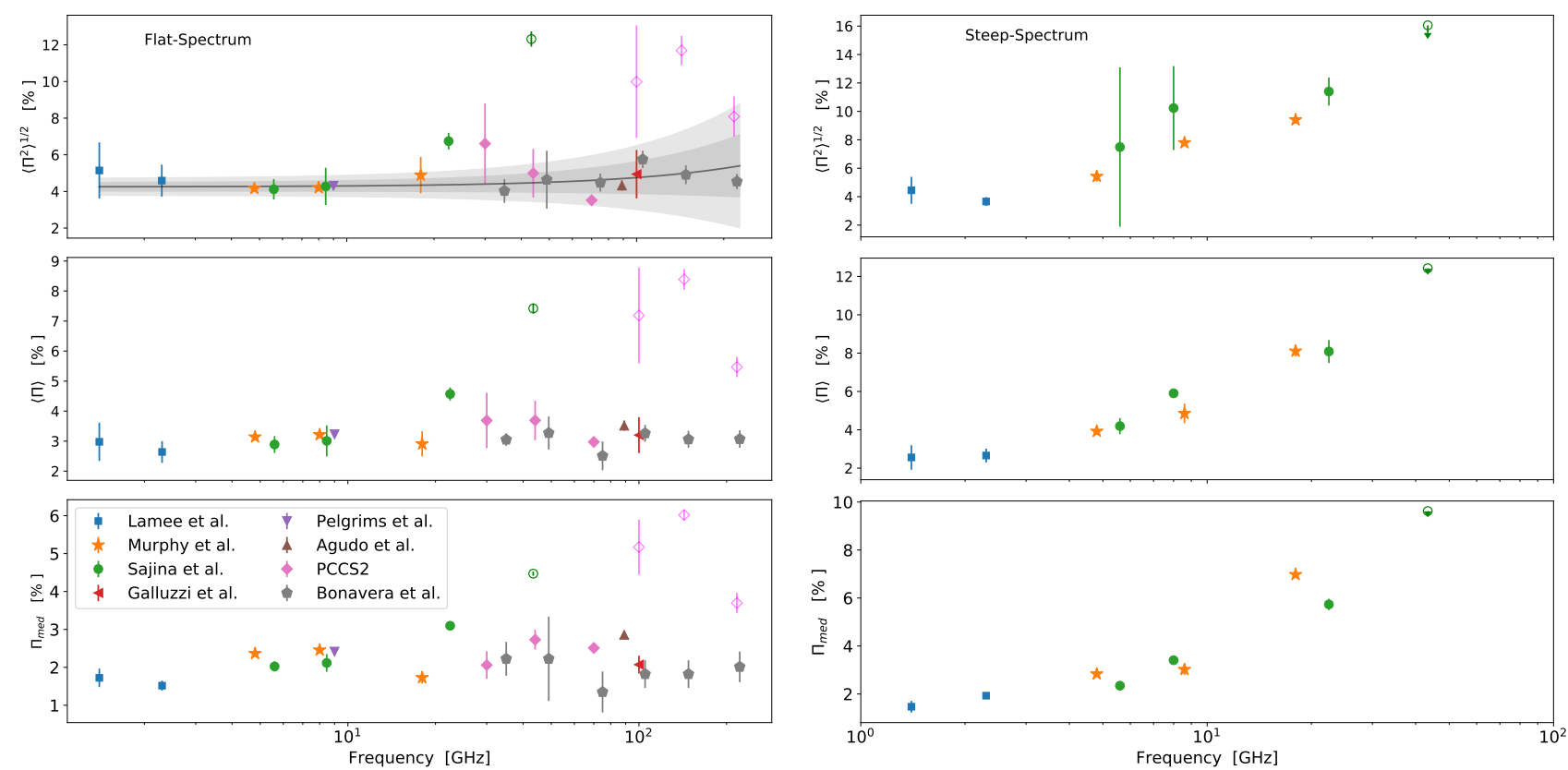

FIG. 3.- Values of $\left\langle\Pi^{2}\right\rangle^{1 / 2}$ (top), $\langle\Pi\rangle$ (center) and $\Pi_{\text {med }}$ (bottom) derived from best fit lognormal parameters (4), 3., 5). Open symbols are data which have not been taken into account for the linear fit in (6). We distinguished FSRQs (left) from the SSRQs (right). In top left panel, a linear function is fitted from the data to provide a scaling of polarization fraction as a function of frequency. Light and dark shaded area are respectively the $1 \sigma$ and $2 \sigma$ uncertainties.

TABLE 3

NOMinal SPECIFICS OF CMB EXPERIMENTS DESCRIBED IN SEC 5

\begin{tabular}{lcccc}
\hline & Frequency $[\mathrm{GHz}]$ & Sensitivity $[\mu \mathrm{K}$ arcmin $]$ & FWHM & $f_{\text {sky }}$ \\
\hline QUIJOTE & $11,13,17,19$ & 1800 & $1^{\circ}$ & $50 \%$ \\
CMB-S2 & 95,150 & 25,30 & $3.5^{\prime}$ & $5 \%$ \\
CMB-S3 SA & $30,40,95,150$ & $8,6,1,2$ & $1^{\circ}$ & $20 \%$ \\
CMB-S3 LA & $30,40,95,150$ & $8,6,1,2$ & $10^{\prime}, 7^{\prime}, 3^{\prime}, 2^{\prime}$ & $20 \%$ \\
LiteBIRD & $40,50,60,68,78$ & $53,32,25,19,15$ & $1^{\circ}$ & $100 \%$ \\
& $89,100,119,140,166$ & $12,15.6,12.6,8.3,8.7$ & $1^{\circ}$ & $100 \%$ \\
CORE150 & $60,100,145$ & $10.6,7.1,5.1$ & $14^{\prime}, 8^{\prime}, 6^{\prime}$ & $100 \%$ \\
\hline
\end{tabular}

TABLE 4

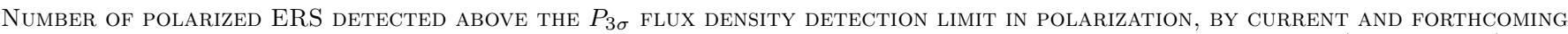
CMB ground based experiments. Counts are estimated both from the D05 and the C2Ex predictions (in Brackets).

\begin{tabular}{lcc|cccc}
\hline & \multicolumn{2}{c}{ CMB -S2 } & \multicolumn{4}{c}{ CMB -S3 } \\
$\nu[\mathrm{GHz}]$ & $P_{3 \sigma}[\mathrm{mJy}]$ & $N_{3 \sigma}$ & $P_{3 \sigma}[\mathrm{mJy}]$ & $N_{3 \sigma}$ & $P_{3 \sigma}[\mathrm{mJy}]$ & $N_{3 \sigma}$ \\
\hline 30 & $\cdots$ & $\cdots$ & 15 & $236(191)$ & 1.5 & $2329(2278)$ \\
40 & $\ldots$ & $\ldots$ & 15 & $215(156)$ & 1.5 & $1867(1810)$ \\
95 & 100 & $3(2)$ & 10 & $355(222)$ & 1 & $2432(2136)$ \\
150 & 100 & $3(1)$ & 15 & $146(74)$ & 1.5 & $1145(867)$ \\
\hline
\end{tabular}

$S_{c u t}=3 \sigma_{\text {det }}$ and we do not include them into power spectrum estimate

$$
C_{\ell}^{T}=\left(\frac{d B}{d T}\right)^{-2} N\left\langle S^{2}\right\rangle=\left(\frac{d B}{d T}\right)^{-2} \int_{0}^{S_{c u t}} n(S) S^{2} d S
$$

where $n(S)$ and $N$ are respectively the differential and the integral number counts per steradian, and $d B / d T$ is the conversion factor from brightness to temperature, being

$$
\left(\frac{d B}{d T}\right)^{-1} \approx 10^{-2} \frac{\left(e^{x}-1\right)^{2}}{x^{4} e^{x}} \frac{\mu \mathrm{K}}{\mathrm{Jy} \mathrm{sr}^{-1}}
$$

with $x=\nu / 57 \mathrm{GHz}$. Tucci et al. (2004) found that it possible to relate the ERS polarization power spectrum to the intensity one (7) as follows

$$
\begin{aligned}
C_{\ell}^{Q} & =\quad\left(\frac{d B}{d T}\right)^{-2} N\left\langle Q^{2}\right\rangle \\
& =\left(\frac{d B}{d T}\right)^{-2} N\left\langle S^{2} \Pi^{2} \cos ^{2} 2 \phi\right\rangle \\
& =\left(\frac{d B}{d T}\right)^{-2} N\left\langle S^{2}\right\rangle\left\langle\Pi^{2}\right\rangle\left\langle\cos ^{2} 2 \phi\right\rangle \\
& =\quad \frac{1}{2}\left(\frac{d B}{d T}\right)^{-2}\left\langle\Pi^{2}\right\rangle C_{\ell}^{T},
\end{aligned}
$$

where the $1 / 2$ factor comes from the average value of $\cos ^{2} 2 \phi$, if the polarization angle $\phi$ is uniformly dis- 
TABLE 5

Number of Sources Detected ABove the $\geq S_{\text {lim }}$ AND $\geq P_{\text {lim }}$ FLUX DENSITIES LIMIT BY THE QUIJOTE EXPERIMENT, ASSUMING THE NOMINAL AND CONSERVATIVE VALUES FOR SENSITIVITY. VAlues ARE ESTIMATED USING D05 AND C2Ex MODELS (INS BRACKETS).

\begin{tabular}{lcccc}
\hline$\nu[\mathrm{GHz}]$ & $S_{\text {lim }}[\mathrm{Jy}]$ & $N_{\text {src }}$ & $P_{\text {lim }}[\mathrm{Jy}]$ & $N_{\text {src }}$ \\
\hline \multirow{2}{*}{11} & 0.5 & $694(673)$ & 0.5 & $6(4)$ \\
& 1 & $347(340)$ & 1 & $2(1)$ \\
13 & 0.5 & $445(434)$ & 0.5 & $2(1)$ \\
& 1 & $210(205)$ & 1 & $0(0)$ \\
17 & 1 & $201(197)$ & 1 & $0(0)$ \\
& 2 & $86(83)$ & 2 & $0(0)$ \\
19 & 1 & $128(125)$ & 1 & $0(0)$ \\
& 2 & $52(51)$ & 2 & $0(0)$ \\
\hline
\end{tabular}

TABLE 6

NUMBER OF SOURCES OBSERVED ABOVE $3 \sigma_{\text {det }}$ LIMIT IN TERMS OF POLARIZED FLUX DENSITY $P_{3} \sigma$ BY THE LITEBIRD EXPERIMENT. Bracketed VAlues are estimated using the C2Ex model.

\begin{tabular}{lcc}
\hline$\nu[\mathrm{GHz}]$ & $P_{3 \sigma}[\mathrm{mJy}]$ & $N_{3 \sigma}$ \\
\hline 40 & 450 & $4(3)$ \\
50 & 240 & $11(8)$ \\
60 & 210 & $9(6)$ \\
68 & 300 & $4(3)$ \\
78 & 240 & $6(4)$ \\
89 & 210 & $12(8)$ \\
100 & 240 & $10(7)$ \\
119 & 210 & $14(10)$ \\
140 & 270 & $8(4)$ \\
166 & 270 & $7(4)$ \\
\hline
\end{tabular}

tributed. The value for $\left\langle\Pi^{2}\right\rangle$ is derived at each frequency from eq. (6). Since we do expect point sources to equally contribute on average both to $Q$ and $U$, and thus to the $E$ - and $B$ - modes, we can approximate $C_{\ell}^{B} \simeq C_{\ell}^{E} \simeq C_{\ell}^{U} \simeq C_{\ell}^{Q}$. In the following, B-mode power spectra are normalized by the usual normalization factor $\mathcal{D}_{\ell}=\ell(\ell+1) C_{\ell} / 2 \pi$.

To forecast the number of sources that will be detected in intensity and polarized flux-density above a given detection limit, we integrate the differential number counts, $n(S)$ and $n(P)$ as

$$
\begin{aligned}
& N(>S)=\int_{S_{c u t}}^{\infty} n(S) d S, \\
& N(>P)=\int_{P_{\text {cut }}}^{\infty} n(P) d P .
\end{aligned}
$$

Finally, to compare the level of contamination produced by the ERS with the Galactic foreground one, we rescale the Galactic foreground emission at a given $f_{\text {sky }}$, frequency $\nu$ and multipole order $\ell$ as in Planck Collaboration et al. (2016b),

$$
\begin{aligned}
\mathcal{D}^{F G}\left(\ell, \nu, f_{s k y}\right)= & \frac{\operatorname{Var}\left[\text { Sync, } f_{\text {sky }}\right]}{\operatorname{Var}\left[\text { Sync, } f_{\text {sky }, 0}\right]} q_{s}\left(\frac{\ell}{80}\right)^{\alpha_{s}} \frac{s_{s}(\nu)}{s_{s}\left(\nu_{s}\right)}+ \\
& \frac{\operatorname{Var}\left[\text { Dust }, f_{\text {sky }}\right]}{\operatorname{Var}\left[\text { Dust, } f_{\text {sky }, 0}\right]} q_{d}\left(\frac{\ell}{80}\right)^{\alpha_{d}} \frac{s_{d}(\nu)}{s_{d}\left(\nu_{d}\right)} .
\end{aligned}
$$

with $s, d$ referring respectively to synchrotron and dust. For all the parameters entering in (11), we use the best fit values quoted in Planck Collaboration et al. (table 11 2016b) estimated outside the Galactic plane in the
UPB77 mask (Planck Collaboration et al. 2016a, defined in section 4.2). The mask has been computed considering a common foreground mask after component separation analysis with $1^{\circ}$ apodization scale. Therefore, to rescale the estimate in eq. 11) to a patch with a smaller fraction of sky, $f_{s k y}$, we need to compute the variance of both synchrotron and thermal dust template maps within the considered patch and within the Planck region with $f_{s k y, 0}=73 \%$. The rescaled foreground power spectra are shown in Figure 4 as dotted lines.

\section{1. $P S_{4} C$ with current and forthcoming $C M B$ ground based experiments}

Figure 4 shows our PS4C forecasts of foreground contamination to the recovery of the CMB B-mode for the different experiments in the different panels: we plot the expected spectrum in polarization of Galactic (dotted lines) and ERS (dashed lines) emissions at the different frequencies available for each experiment and the total CMB B-mode power spectrum (black solid line). The black dot-dashed lines show the primordial $(r=0.05)$ and lensed B-mode power spectra separately. The power spectra are computed in the region outside the UPB77 Planck mask (in order to exclude the Galactic plane and the ERS whose flux density is below the $3 \sigma$ detection limit). The Galactic foreground turns out to be the most contaminating emission in the B-mode recovery. The different colors for the Galactic and ERS spectra are for different frequencies, going from purple to yellow as the frequency increases. It should be commented that there exists several component separation and foreground cleaning algorithms that can recover CMB intensity and polarization signals with great accuracy (Planck Collaboration et al. 2016b). In addition, multi-frequency observations and joint analyses from different experiments (BICEP2/Keck and Planck Collaborations et al. 2015) can improve the foreground cleaning. So, even if in our work we are considering the most conservative cases, it should be stressed that such contamination could be lowered ( at sub-percentage level Stompor et al. 2016, Errard et al. 2011) by applying such foreground removal algorithms.

In particular, Figure 4 shows our forecasts for the QUIJOTE (top left) and CMB-S2 (top right) experiments. As for QUIJOTE, the Galactic emission is much higher than the CMB one and higher than the contribution from undetected ERS, except at small angular scales where the ERS start to be dominant. Since the QUIJOTE experiment ranges from 10 to $20 \mathrm{GHz}$, we need to take into account the contribution from both FSRQs and SSRQs, with the resulting increase in the average fractional polarization and number counts (see Figure 3 and Figure A.1. Table 5 summarizes the total number of sources in total intensity (third column) and polarization (fourth column) that QUIJOTE would detect (frequencies are given in the first column), assuming nominal and conservative sensitivity values (flux density limits in total intensity and polarization are listed in columns two and three respectively). We found $694,445,201$ and 128 sources in total intensity at $11,13,17,19 \mathrm{GHz}$ respectively. In polarization only a few of them would be detected and just in the 11 and $13 \mathrm{GHz}$ channels.

For the CMB-S2 experiment whose frequencies are greater than $95 \mathrm{GHz}$, the Galactic emission (mostly ther- 

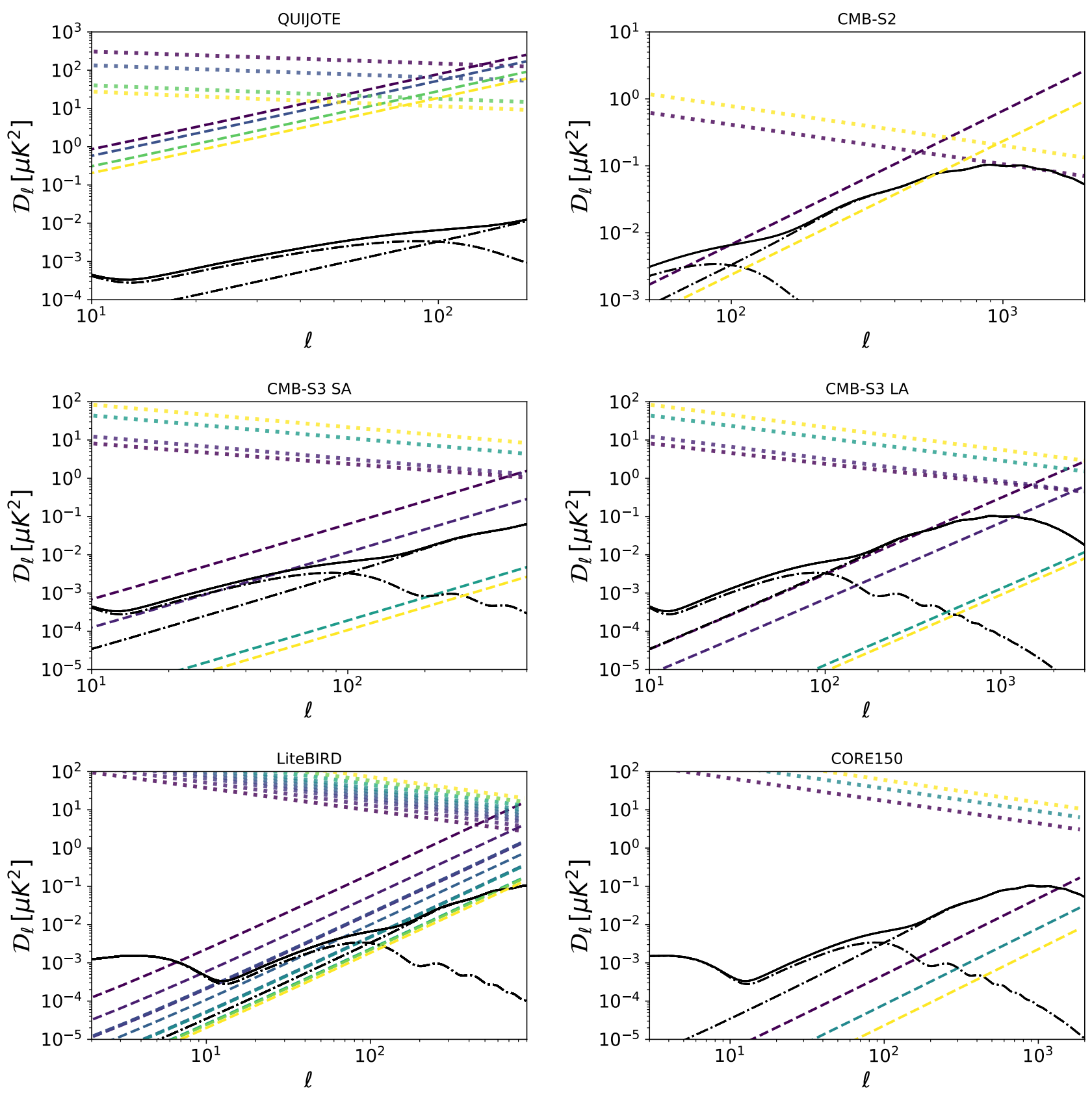

FIG. 4.- Forecasts of foreground contamination with PS4C. In all panels, the black dot-dashed lines show the primordial $(r=0.05)$ and lensed CMB B-mode power spectra and the black solid line is the the total CMB B-mode power spectrum. The dotted (dashed) lines are the power spectrum of the polarized Galactic emission (ERS emission) at the different frequencies available for each experiment, the color scale is such that the colors go from purple to yellow as the frequency increases. The power spectra depend are estimated using eq. 11 in the region outside the UPB77 Planck mask (in order to exclude the Galactic plane and ERS above the $3 \sigma$ detection limit). The different panels corresponds to predictions for different experiments. From top to bottom and from left to right: QUJOTE (11, 13, 17, 19 GHz), CMB-S2 (95 and $150 \mathrm{GHz}$ ), CMB-S3 observing with small and large aperture telescopes (30, 40, 95, 150 GHz), LiteBIRD (frequencies between $40-166 \mathrm{GHz})$ and CORE150 $(60,100,145 \mathrm{GHz}$. 
mal dust emission) is the most contaminating up to $\ell \sim 350$, while the ERS are important at small angular scales. Unlike the previous case, at these frequencies the CMB B-mode spectrum is comparable to the one of undetected ERS.

In Figure 5 the triangles show the $C_{\ell}^{B B}$ of undetected ERS estimated using eq. (8). The detection limits are given by the CMB-S2 sensitivities. The $C_{\ell}^{B B}$ of the CMB B-mode are also plotted: the cyan dashed line is for the case $\ell \approx 80$ and $r=0.05$ and the orange dashed line is for $\ell \approx 1000$. Figure 5 shows what is the contamination due to undetected ERS and consequently the level of source detection required to detect primordial or lensing $B$-mode signal. In CMB-S2 the undetected ERS level of the power spectrum is comparable to the lensing Bmode one. In this case, given the experiment sensitivity and the size of the observed region, $\sim 150$ sources would be detected in total intensity and only few of them in polarization at a $3 \sigma$ level.

Among the experiments studied in this work, the CMB-S3 is the one with the greatest sensitivity and best resolution. The results are shown in the central panels of Figure 4 and in the left panel of Figure 5 with circles and diamonds. As summarized in Table 4, the maximum number of polarized sources detected above a $3 \sigma$ level and using the large aperture telescope is 2329 with flux density $P_{\text {lim }} \gtrsim 1 \mathrm{mJy}$. When using a smaller aperture telescope, this number drops to a few hundreds with polarized flux densities $P_{\text {lim }} \gtrsim 10 \mathrm{mJy}$.

The contribution in polarization of undetected ERS is very small at high frequencies $(\nu \gtrsim 90)$ and at low multipoles $\ell \lesssim 2000$. At lower frequencies, undetected ERS still can contaminate and they have to be taken into account to de-lens, lensing $B$-modes to get the primordial ones for $r \lesssim 0.05$.

\subsection{PS4C with future space missions}

The results for the LiteBIRD experiment are shown in the left bottom panel of Figure 4 and the filled circles in the right panel of Figure 5. On the whole, the most contaminating contribution is the Galactic one, except at small angular scales $(l \sim 400)$ and high frequencies $(\nu>70 \mathrm{GHz})$ where the ERS contribution is comparable to the Galactic one. The ERS contribution, although generally lower than the Galactic one, is also important being higher than the CMB B-mode level even at large scales $(l \gtrsim 7)$ and $\nu<70 \mathrm{GHz}$ (dashed purple and blue lines). Moreover, at $\nu>80 \mathrm{GHz}$ and $l \gtrsim 70$ the ERS contribution is comparable to the B-mode power spectrum. The number of sources that would be detected in polarization above the $3 \sigma$ level with this experiment are listed in Table 6 and they range from 4 at 10 and 68 $\mathrm{GHz}$ to 14 at $119 \mathrm{GHz}$. The first column is the frequency in $\mathrm{GHz}$, the second is the polarized flux density limit in mJy and the third column is the number of sources that would be detected by LiteBIRD (values in the brackets are estimated from the C2Ex model).

Our findings for CORE are shown in the right bottom panel of Figures 4 and in the right panel of Figure 5 (squares). Galactic emission is the most contaminating for B-mode detection. Undetected ERS are important only at $60 \mathrm{GHz}$, where their power spectrum is comparable to the one of the B-mode due to lensing. CORE would be able to detect up to 200 sources per steradian, implying a lower contamination for the CMB B-mode power spectrum with respect to LiteBIRD.

Table 7 compares the surface densities (i.e. number of sources per steradian, last two columns) at CORE frequencies (first column) of the polarized ERS above the $P_{4 \sigma}$ flux density limit (second column) estimated by De Zotti et al. (2016)(DZ16) and PS4C (values in the brackets are for C3Ex estimate). In this comparison we use a $4 \sigma$ flux density limit in order to be consistent with the estimates by De Zotti et al. (2016). Above $100 \mathrm{GHz}$, we find a discrepancy between D05 and DZ16 that could be due to two effects that become more important at higher frequencies: (i) the D05 predictions tend to overestimate the polarized source number counts (see Sec. 3 ) and (ii) at $\nu>100$ the polarization fraction is expected to suffer a slight increase (from $\sim 4 \%$ to $\sim 5 \%$ from 100 to $150 \mathrm{GHz}$ ) as can be seen in eq. 60 and Figure 3 .

On one hand, at $100 \mathrm{GHz}$, we find that accounting solely for the observation in (ii), i.e. a $20 \%$ increase of $\Pi$ to a value of $4.67 \%$, the D05 forecasts predict source counts that are $20 \%$ larger than DZ16 ${ }^{13}$. On the other hand, at $150 \mathrm{GHz}$, the surface density estimated with PS4C with D05 model is $\sim 65 \%$ larger than the value referred by DZ16. Even accounting for the $25 \%$ fractional increase of $\Pi$ to $4.92 \%$ from eq.(6), this is not enough to compensate the observed discrepancy. We thus argue that the discrepancy at $150 \mathrm{GHz}$ is caused by both (i) and (ii).

Contrary to the D05 forecasts, the C2Ex model is in reasonable agreement with De Zotti et al. (2016), meaning that the C2Ex predictions are more robust than the D05 ones at least at higher frequencies.

TABLE 7

COMPARISON OF SURFACE DENSITIES OF POLARIZED ERSS BRIGHTER THAN $P_{4 \sigma}$ ESTIMATED By De ZotTI ET AL. 2016)(DZ16) AND BY PS4C. VAlues in BRACKETS REFER to C2EX ESTIMATES.

\begin{tabular}{lccc}
\hline$\nu[\mathrm{GHz}]$ & $P_{4 \sigma}[\mathrm{mJy}]$ & \multicolumn{2}{c}{$N_{4 \sigma}\left[\mathrm{sr}^{-1}\right]$} \\
& & DZ16 & PS4C \\
\hline 60 & 5.2 & 212 & $214(198)$ \\
100 & 5.2 & 184 & $229(164)$ \\
145 & 4.6 & 165 & $271(142)$ \\
\hline
\end{tabular}

\section{SUMMARY AND CONCLUSIONS}

We describe and present the state-of-the-art observations on polarization of ERS over a wide frequency range, namely from 1.4 to $217 \mathrm{GHz}$. We exploit for the first time the polarization number counts at $95 \mathrm{GHz}$ from a sample of 32 polarized sources detected with ALMA. The characterization of these sources and their spectral behaviour in frequencies ranging from 1 to $95 \mathrm{GHz}$ are described in a companion paper by Galluzzi et al. (2018, in prep.)

By collecting polarization flux densities from 10 catalogues, we are able to derive a relation of the average fractional polarization as a function of frequency and to avoid extrapolations that have been commonly adopted to forecast the average polarization fraction from low( $\lesssim 20 \mathrm{GHz}$ where enough data have been collected), to high-frequency $(\gtrsim 70 \mathrm{GHz}$ where still few polarization

13 For this estimate, we assume that differential source counts are described by a power law with spectral index $>1$ 

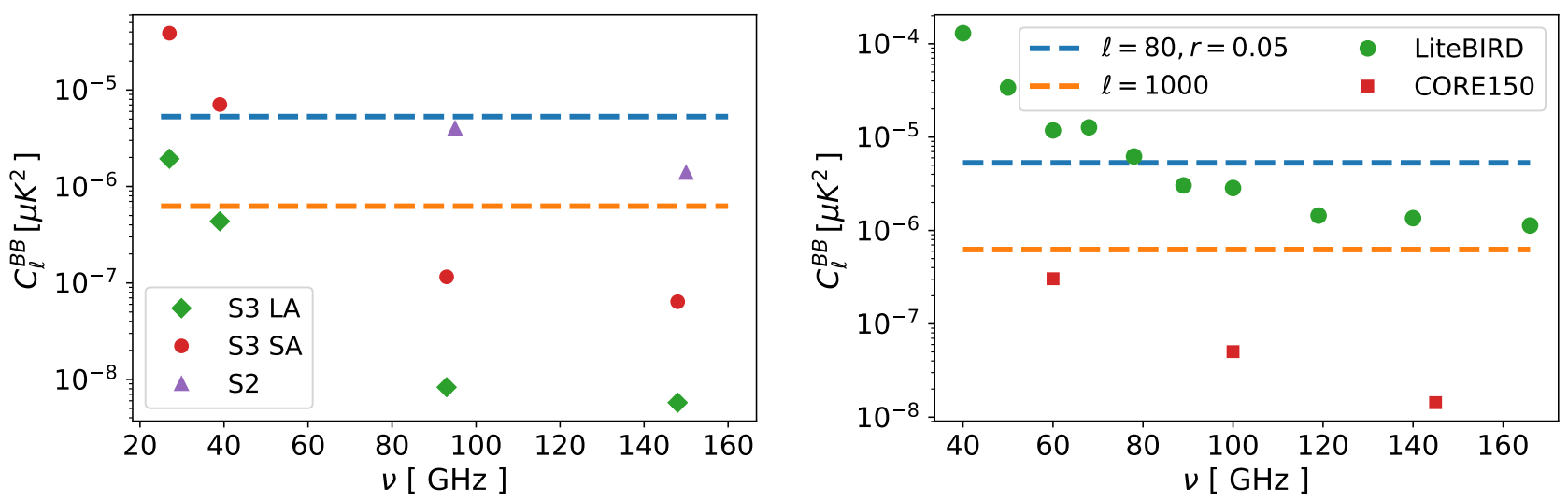

FIG. 5.- Power spectra in polarization of undetected ERS in current and future CMB experiments. Left panel: CMB-S2 (triangles) and CMB-S3 (circles for the small aperture telescope and diamonds for the large aperture telescope). Right panel: LiteBIRD (circles) and CORE150 (squares). The dotted lines are the B-mode power spectra at the acoustic scale $(\ell=80)$ and at the lensing B-modes peak scale $(\ell \approx 1000)$.

measurements have been performed). Therefore, we fit a linear function on data from several surveys, including Planck measurements from both detection and stacking, and we find a mild dependence of $\left\langle\Pi^{2}\right\rangle^{1 / 2}$ as a function of $\nu$.

This relation allows us to forecasts the contribution of ERSs to polarization B-mode power spectrum given the nominal sensitivities of current and forthcoming $\mathrm{CMB}$ experiments, by means of predictions of ERS counts coming from two models D05 and C2Ex. The whole forecast suite is fully integrated into a Python package, PS4C, made publicly available with online documentation and tutorials.

We discuss the reasons why we do not assume a correlation between the level of fractional polarization and the total intensity flux. Although still controversial and not observed at high-radio frequencies (Galluzzi et al. 2018 in prep., Galluzzi et al. 2018: Galluzzi et al. 2017; Massardi et al. 2013), deeper surveys in polarization are critical to further proof the validity of this assumption, not only at higher frequencies but also at fainter flux density levels.

Future CMB experiments could shed light on this interesting aspect: in fact, we have shown that they are going to observe an increasing number of polarized ERS (they are foreseen to detect up to $\sim 2000$ polarized ERS) because their sensitivity will increasingly improve in the future.

A further potentiality of future $\mathrm{CMB}$ experiments is that they can be largely exploited by the community as wide global surveys to measure polarized flux density of sources at very high-radio frequencies (Partridge et al. 2017). Programs aimed at observing ERSs at higher resolution can thus benefit of CMB large area surveys in an extremely wide range of frequencies, from 20 up to 300 $\mathrm{GHz}$.

Moreover, since in this work we mostly focus on blazar statistical polarization, as it is the main bright source population at frequencies $<150 \mathrm{GHz}$, we restrict our forecast analysis up to this frequency limit. At higher frequencies, the far-IR dusty star forming galaxies constitute the majority of extra-galactic sources (see Planck Collaboration (fig.25 2015)) and, similarly to the ERSs, their polarized emission contaminates B-mode power spectra ${ }^{14}$ (De Zotti et al. 2015). Recent works from Bonavera et al. (2017b); De Zotti et al. (2016) have already shown statistical polarization properties of dusty sources and forecasted their contribution for future CMB experiments. Therefore, we plan to include those estimates within the PS4C package in a future development.

As a final remark, we stress that ERSs below the detection flux limit may introduce a bias at all the angular scales and at frequencies $\nu<50 \mathrm{GHz}$ : their synchrotron emission is still strong enough to contaminate polarization measurements even at low flux densities, namely $P<1$ mJy. At larger frequencies, ERS polarization power spectra have to be assessed as long as smaller angular scales are involved to estimate the $\mathrm{CMB}$ power spectrum at multipoles around the lensing peak or to estimate the primordial B-mode power spectrum at lower multipoles $(\ell<800)$ by means of de-lensing algorithms.

Acknowledgments. We are pleased to thank Matteo Bonato, Bruce Partridge, Vincent Pelgrims and the referee for their helpful comments and suggestions. This work was supported by the RADIOFOREGROUNDS grant of the European Union's Horizon 2020 research and innovation programme (COMPET-05-2015, grant agreement number 687312) and by by National Institute of Nuclear Physics (INFN) INDARK. LB and JGN acknowledge financial support from the I+D 2015 project AYA2015-65887-P (MINECO/FEDER). J.G.N also acknowledges financial support from the Spanish MINECO for a Ramon y Cajal fellowship (RYC-2013-13256). VG and MM acknowledge financial support by the Italian Ministero dell'Istruzione, Università $e$ Ricerca through the grant Progetti Premiali 2012-iALMA (CUP C52I13000140001). We further acknowledge support from the ASI-COSMOS Network

\section{REFERENCES}


Abazajian, K. N., Adshead, P., Ahmed, Z., et al. 2016, ArXiv e-prints, arXiv: 1610.02743

Agudo, I., Thum, C., Wiesemeyer, H., \& Krichbaum, T. P. 2010, The Astrophysical Journal Supplement Series, 189, 1

Arnold, K., Stebor, N., Ade, P. A. R., et al. 2014, in Proc. SPIE, Vol. 9153, Millimeter, Submillimeter, and Far-Infrared Detectors and Instrumentation for Astronomy VII, 91531F

Austermann, J. E., Aretxaga, I., Hughes, D. H., et al. 2009, MNRAS, 393, 1573

Battye, R. A., Browne, I. W. A., Peel, M. W., Jackson, N. J., \& Dickinson, C. 2011, MNRAS, 413, 132

Benson, B. A., Ade, P. A. R., Ahmed, Z., et al. 2014, in Proc. SPIE, Vol. 9153, Millimeter, Submillimeter, and Far-Infrared Detectors and Instrumentation for Astronomy VII, 91531P

BICEP2/Keck and Planck Collaborations, Ade, P. A. R. Aghanim, N., et al. 2015, Phys. Rev. Lett., 114, 101301

Blandford, R. D., \& Koenigl, A. 1979, Astrophys. Lett., 20, 15

Bonaldi, A., Bonavera, L., Massardi, M., \& De Zotti, G. 2013, MNRAS, 428, 1845

Bonato, M., Negrello, M., Mancuso, C., et al. 2017, Monthly Notices of the Royal Astronomical Society, Volume 469, Issue 2, p.1912-1923, 469, 1912

Bonavera, L., González-Nuevo, J., Argüeso, F., \& Toffolatti, L. 2017a, MNRAS, 469, 2401

Bonavera, L., González-Nuevo, J., De Marco, B., Argüeso, F., \& Toffolatti, L. 2017b, MNRAS, 472, 628

Bonavera, L., Massardi, M., Bonaldi, A., et al. 2011, MNRAS, 416,559

Condon, J. J., Cotton, W. D., Greisen, E. W., et al. 1998, The Astronomical Journal, 8065, 1693

Curto, A., Tucci, M., González-Nuevo, J., et al. 2013, MNRAS, 432, 728

De Zotti, G., Massardi, M., Negrello, M., \& Wall, J. 2010, Radio and millimeter continuum surveys and their astrophysical implications, 0908.1896

de Zotti, G., Ricci, R., Mesa, D., et al. 2005, A\&A, 431, 893

De Zotti, G., Castex, G., González-Nuevo, J., et al. 2015, JCAP, 6,018

De Zotti, G., Gonzalez-Nuevo, J., Lopez-Caniego, M., et al. 2016, ArXiv e-prints, arXiv:1609.07263

Delabrouille, J., de Bernardis, P., Bouchet, F. R., et al. 2017, ArXiv e-prints, arXiv:1706.04516

Errard, J., Stivoli, F., \& Stompor, R. 2011, Phys. Rev. D, 84, 069907

Galluzzi, V., \& Massardi, M. 2016, International Journal of Modern Physics D, 25, 1640005

Galluzzi, V., Massardi, M., Bonaldi, A., et al. 2017, Monthly Notices of the Royal Astronomical Society, 465, 4085

Galluzzi, V., Massardi, M., Bonaldi, A., et al. 2018, MNRAS, 475, 1306

Gehrels, N. 1986, The Astrophysical Journal, 303, 336

Goldreich, P., \& Kylafis, N. D. 1981, The Astrophysical Journal, $243, \mathrm{~L} 75$

González-Nuevo, J., Toffolatti, L., \& Argüeso, F. 2005, ApJ, 621,

Guth, A. H. 1981, Phys. Rev. D, 23, 347

Henderson, S. W., Allison, R., Austermann, J., et al. 2016, Journal of Low Temperature Physics, 184, 772

Hu, W., \& White, M. J. 1997, New Astron., 2, 323

Jackson, N., Battye, R. A., Browne, I. W. A., et al. 2007, Monthly Notices of the Royal Astronomical Society, 376, 371

Jackson, N., Browne, I. W. A., Battye, R. A., Gabuzda, D., \& Taylor, A. C. 2010, MNRAS, 401, 1388

Keisler, R., Hoover, S., Harrington, N., et al. 2015, The Astrophysical Journal, 807, 151

Kellermann, K. I. 1966, ApJ, 146, 621

Lamee, M., Rudnick, L., Farnes, J. S., et al. 2016, ApJ, 829, 5

Lewis, A., Challinor, A., \& Lasenby, A. 2000, Astrophys. J., 538, 473

Lopez-Caniego, M., Massardi, M., Gonzalez-Nuevo, J., et al. 2009, The Astrophysical Journal, Volume 705, Issue 1, pp 868-876 (2009)., 705, 868

López-Caniego, M., Rebolo, R., Aguiar, M., et al. 2014, ArXiv e-prints, arXiv:1401.4690
Louis, T., Grace, E., Hasselfield, M., et al. 2017, JCAP, 6, 031 Mancuso, C., Lapi, A., Prandoni, I., et al. 2017, ApJ, 842, 95 Marriage, T. A., Baptiste Juin, J., Lin, Y.-T., et al. 2011, ApJ, 731,100

Massardi, M., Bonaldi, A., Bonavera, L., et al. 2011, MNRAS, 415,1597

Massardi, M., López-Caniego, M., González-Nuevo, J., et al. 2009, MNRAS, 392, 733

Massardi, M., Ekers, R. D., Murphy, T., et al. 2008, Mon. Not. R. Astron. Soc, 384, 775

-. 2011, Monthly Notices of the Royal Astronomical Society, 412,318

Massardi, M., Burke-Spolaor, S. G., Murphy, T., et al. 2013, Monthly Notices of the Royal Astronomical Society, 436, 2915

Matsumura, T., Akiba, Y., Arnold, K., et al. 2016, Journal of Low Temperature Physics, 184, 824

Mocanu, L. M., Crawford, T. M., Vieira, J. D., et al. 2013, ApJ, 779,61

Murphy, T., Sadler, E. M., Ekers, R. D., et al. 2010, Monthly Notices of the Royal Astronomical Society, 402, 2403

Negrello, M., Clemens, M., Gonzalez-Nuevo, J., et al. 2013, MNRAS, 429, 1309

Partridge, B., Bonavera, L., López-Caniego, M., et al. 2017 , Galaxies, 5, 47

Pelgrims, V., \& Hutsemékers, D. 2015, Monthly Notices of the Royal Astronomical Society, 450, 4161

Planck Collaboration. 2015, Astronomy \& Astrophysics, 594, A26

Planck Collaboration, Ade, P. A. R., Aghanim, N., et al. 2011a, Astronomy \& Astrophysics, 536, A13

Planck Collaboration, Aatrokoski, J., Ade, P. A. R., et al. 2011b, Astronomy \& Astrophysics, 536, A15

Planck Collaboration, Ade, P. A. R., Aghanim, N., et al. 2013 Astronomy \& Astrophysics, 550, A133

Planck Collaboration, Adam, R., Ade, P. A. R., et al. 2016a, A\&A, 594, A9

- 2016b, A\&A, 594, A10

Planck Collaboration, Ade, P. A. R., Aghanim, N., et al. 2016c, A\&A, 594, A13

Planck Collaboration, Ade, P. A. R., Aghanim, N., et al. 2016d, Astronomy \& Astrophysics, 594, A25

Planck Collaboration, Adam, R., Ade, P. A. R., et al. 2016e, A\&A, 586, A133

Puglisi, G., Fabbian, G., \& Baccigalupi, C. 2017, MNRAS, 469, 2982

Sajina, A., Partridge, B., Evans, T., et al. 2011, The

Astrophysical Journal, 732, 45

Seljak, U., \& Zaldarriaga, M. 1997, Physical Review Letters, 78, 2054

Starobinsky, A. A. 1982, Physics Letters B, 117, 175

Stil, J. M., Keller, B. W., George, S. J., \& Taylor, A. R. 2014, The Astrophysical Journal, 787, 99

Stompor, R., Errard, J., \& Poletti, D. 2016, Phys. Rev. D, 94 083526

The Polarbear Collaboration, Ade, P. A. R., Aguilar, M., et al. 2017, ApJS, 1705.02907

The Polarbear Collaboration: P. A. R. Ade, Akiba, Y., Anthony, A. E., et al. 2014, ApJ, 794, 171

Toffolatti, L., Negrello, M., González-Nuevo, J., et al. 2005, A\&A, 438, 475

Trombetti, T., Burigana, C., De Zotti, G., Galluzzi, V., \& Massardi, M. 2017, ArXiv e-prints, arXiv:1712.08412

Tucci, M., Martínez-González, E., Toffolatti, L., González-Nuevo, J., \& De Zotti, G. 2004, MNRAS, 349, 1267

Tucci, M., \& Toffolatti, L. 2012, Advances in Astronomy, 2012, 624987

Tucci, M., Toffolatti, L., De Zotti, G., \& Martínez-González, E. 2011, Astronomy \& Astrophysics, 533, A57

Vieira, J. D., Crawford, T. M., Switzer, E. R., et al. 2010, The Astrophysical Journal, 719, 763

Waldram, E. M., Pooley, G. G., Davies, M. L., Grainge, K. J. B., \& Scott, P. F. 2010, MNRAS, 404, 1005

Waldram, E. M., Pooley, G. G., Grainge, K. J. B., et al. 2003, MNRAS, 342, 915

Wright, E. L., Chen, X., Odegard, N., et al. 2009, ApJS, 180, 283

\section{APPENDIX}



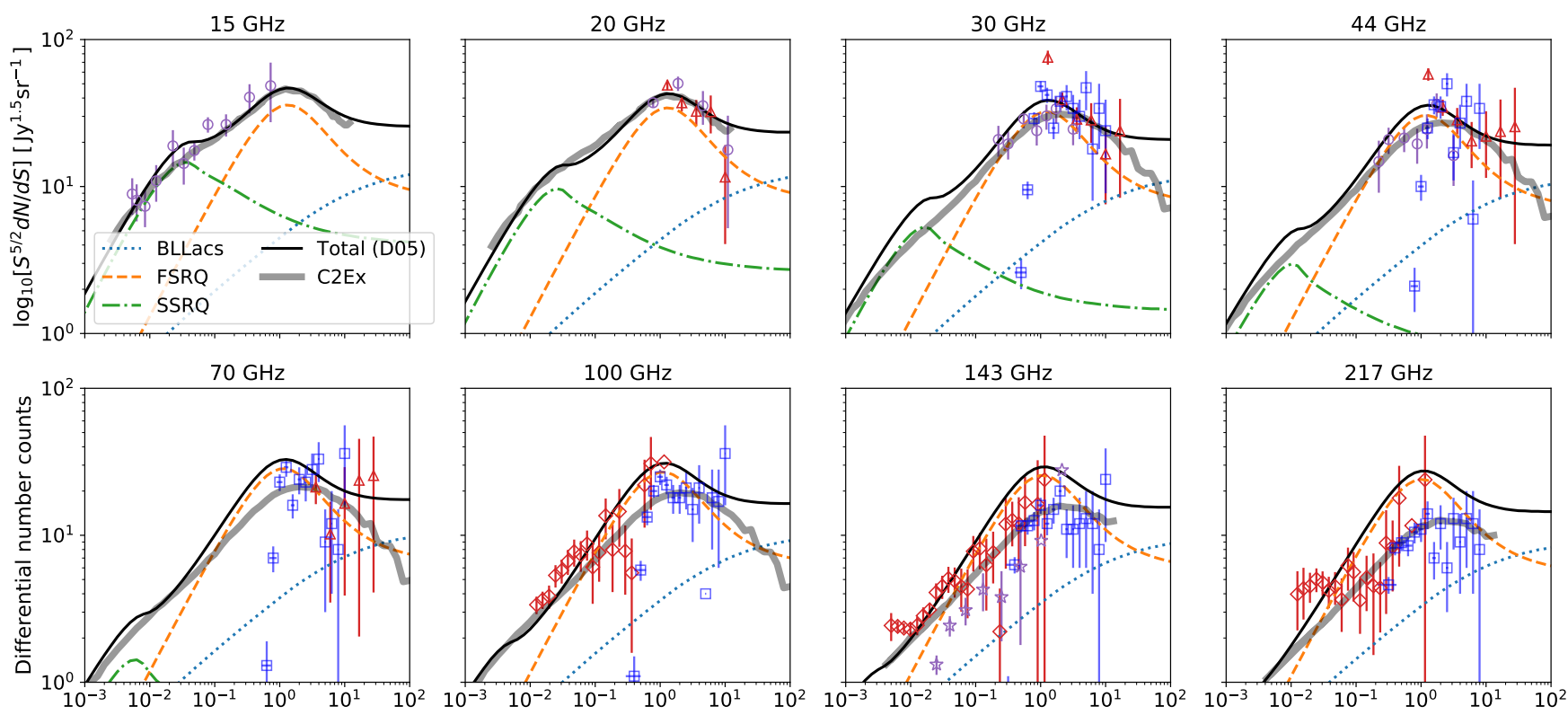

Flux Density $S_{\nu}[J y]$

FIG. A.1. - Euclidean normalized differential number counts on a wide range of frequencies. The dotted, dashed, dot-dashed and solid lines are respectively the number counts of BL Lacs, FSRQs, SSRQs and their total contribution predicted by the D05 model. The thick solid grey line are the number counts coming from the C2Ex model. Number counts obtained with several experiments observing at similar frequency channels are also shown: open circles at 15 and $20 \mathrm{GHz}$ are respectively from Waldram et al. (2003 2010) and Massardi et al. (2008); open circles at 30 and $44 \mathrm{GHz}$ resemble counts from the PACO dataset (Bonavera et al. 2011); upper triangles from 20 to $70 \mathrm{GHz}$ are data from WMAP5-yr survey (Massardi et al. 2009)); diamonds at 100, 143, $217 \mathrm{GHz}$ are from SPT (Mocanu et al. 2013); stars at 143 $\mathrm{GHz}$ are ACT counts (Marriage et al. 2011); squares at 30,44,70,100,143, $217 \mathrm{GHz}$ are based on data from Planck Collaboration et al. (2011a). 